\title{
Self-similar two-particle separation model
}

\author{
Lüthi, Beat; Berg, Jacob; Ott, Søren; Mann, Jakob
}

Published in:

Physics of Fluids

Link to article, DOI:

$10.1063 / 1.2722423$

Publication date:

2007

Document Version

Publisher's PDF, also known as Version of record

Link back to DTU Orbit

Citation (APA):

Lüthi, B., Berg, J., Ott, S., \& Mann, J. (2007). Self-similar two-particle separation model. Physics of Fluids, 19(4), 045110. https://doi.org/10.1063/1.2722423

\section{General rights}

Copyright and moral rights for the publications made accessible in the public portal are retained by the authors and/or other copyright owners and it is a condition of accessing publications that users recognise and abide by the legal requirements associated with these rights.

- Users may download and print one copy of any publication from the public portal for the purpose of private study or research.

- You may not further distribute the material or use it for any profit-making activity or commercial gain

- You may freely distribute the URL identifying the publication in the public portal

If you believe that this document breaches copyright please contact us providing details, and we will remove access to the work immediately and investigate your claim 


\title{
Self-similar two-particle separation model
}

\author{
Beat Lüthi, Jacob Berg, Søren Ott, and Jakob Mann \\ Wind Energy, Ris $\phi$ National Laboratory/DTU, 4000 Roskilde, Denmark
}

(Received 14 July 2006; accepted 8 March 2007; published online 27 April 2007)

\begin{abstract}
We present a new stochastic model for relative two-particle separation in turbulence. Inspired by material line stretching, we suggest that a similar process also occurs beyond the viscous range, with time scaling according to the longitudinal second-order structure function $S_{2}(r)$, e.g.; in the inertial range as $\varepsilon^{-1 / 3} r^{2 / 3}$. Particle separation is modeled as a Gaussian process without invoking information of Eulerian acceleration statistics or of precise shapes of Eulerian velocity distribution functions. The time scale is a function of $S_{2}(r)$ and thus of the Lagrangian evolving separation. The model predictions agree with numerical and experimental results for various initial particle separations. We present model results for fixed time and fixed scale statistics. We find that for the Richardson-Obukhov law, i.e., $\left\langle r(t)^{2}\right\rangle=g \varepsilon t^{3}$, to hold and to also be observed in experiments, high Reynolds numbers are necessary, i.e., $\operatorname{Re}_{\lambda}>\mathcal{O}(1000)$, and the integral scale needs to be large compared to initial separation, i.e., $\mathcal{L} / r_{0}>30$ and $d / \mathcal{L}>3$ need to be fulfilled, where $d$ is the size of the field of view. Removing the constraint of finite inertial range, the model is used to explore separation dynamics in the asymptotic regime. $A s \operatorname{Re}_{\lambda} \rightarrow \infty$, the distance neighbor function takes on a constant shape, almost as predicted by the Richardson diffusion equation. For the Richardson constant we obtain that $g \rightarrow 0.95$ as $\operatorname{Re}_{\lambda} \rightarrow \infty$. This asymptotic limit is reached at $\operatorname{Re}_{\lambda}>1000$. For the Richardson constant $g$, the model predicts a ratio of $g_{b} / g_{f} \approx 1.9$ between backwards and forwards dispersion. () 2007 American Institute of Physics. [DOI: 10.1063/1.2722423]
\end{abstract}

\section{INTRODUCTION}

Relative particle dispersion ${ }^{1}$ is of practical importance to many applied problems in mixing and reactions in industrial as well as environmental flows. It has recently been reviewed by Sawford, ${ }^{2}$ who discussed attempts to evaluate, e.g., the Richardson constant and to exploit the connection between relative dispersion, turbulent mixing, and scalar fluctuations. A few years before that, Shirolkar et al. ${ }^{3}$ extensively reviewed modeling aspects of turbulent particle dispersion. They emphasize dispersion of heavy particles and particle turbulence interaction, as often occurs in practical systems. They stress that fluid particle dispersion in isotropic and homogeneous turbulence is only a subclass of generally more complex flow situations. In, e.g., Refs. 4-6, its relevance also for biological systems has been emphasized. Despite its importance, even relative dispersion of passive fluid particles is still relatively poorly understood. Kinematic simulations (KS) of turbulent-like flows ${ }^{7-9}$ offers the possibility to study a number of dispersion aspects, also at high Reynolds numbers. Only recently ${ }^{10-15}$ has it become possible to study the second moment of the probability density function (PDF) of the separation $r(t)$ between two particles also in real turbulence via direct numerical simulation (DNS) or experiment, with the notable exception of Bourgoin et al. ${ }^{15}$ at typically low to intermediate Reynolds numbers. Relative dispersion of fluid particles still serves as a benchmark problem for our current understanding of turbulence.

Before we outline some specific aspects of the problem, we briefly summarize the current situation in particle dispersion. Existing analytical closures treat particle dispersion as a diffusion problem. ${ }^{1,16-18}$ We note here also the very recent work of Franzese and Cassiani, ${ }^{19}$ who derived a differential equation for the mean square relative separation based on first principles. For a thorough theoretical overview on particle diffusion, we refer to Falkovich et al. ${ }^{20}$ Different assumptions on the diffusion coefficient were made and experiments were conducted in order to evaluate the proposed choices. Recently, particle tracking ${ }^{11,21}$ and numerical ${ }^{14}$ experiments showed that the shape of the separation PDF is approximated best by the Richardson model. This finding was again questioned, though, in Ref. 15. Generally, experiments, both numerical and real, are still limited to flows with low Reynolds numbers. This situation calls for alternative tools to study the influence of such limitations. We mention the eddy-damped quasi-normal Markovian model $($ EDQNM $),{ }^{22,23}$ the Lagrangian-history direct-interaction (LHDI) theory, ${ }^{18}$ kinematic simulations (KS),${ }^{7-9,24}$ and the concept of persistent critical point flow patterns referred to as the statistical persistence hypothesis (SPH) ${ }^{25,26}$ Yet another approach is the class of Lagrangian stochastic models (LSMs). Recent implementations ${ }^{27-29}$ that go back over Thomson $^{30}$ to Durbin ${ }^{31}$ reduce the dispersion problem in a real three-dimensional turbulent flow to a quasi-onedimensional one. They depend upon a detailed knowledge of Eulerian flow statistics, such as acceleration and velocity distributions, which are not easily parametrized. In particular, conditional accelerations statistics are difficult to measure or predict precisely, even though successful measurements are reported by Mordant et al., ${ }^{32}$ Voth et al. ${ }^{33}$ and La Porta et $a l .{ }^{34}$ Our contribution circumvents these difficulties. The presented model depends neither on Eulerian accelerations nor on the precise shape of the Eulerian longitudinal velocity PDF. Instead, it assumes that in the inertial range, a similar 
process to viscous material line stretching occurs, with a governing time scale that evolves in a Lagrangian way as a function of $r(t)$. Thus, we are, similar to Refs. 28 and 29, following Richardson's ${ }^{1}$ assumption that the diffusion coefficient is dependent only on the separation and not on time.

The work of Richardson, ${ }^{1}$ Obukhov, ${ }^{16}$ and Batchelor ${ }^{17}$ led to today's understanding of the ballistic regime, i.e., $\left\langle r^{2}(t)-r_{0}^{2}\right\rangle \propto t^{2}$, and to the $\left\langle r^{2}(t)\right\rangle=g \varepsilon t^{3}$ law. If a Batchelor time is defined as $\tau_{B}=\left(r_{0}^{2} / \varepsilon\right)^{1 / 3}$, the former regime is for $t$ $<\tau_{B}$ and the latter for $\tau_{B}<t<\mathcal{T}$, where $\mathcal{T}$ is the integral time. In the ballistic regime the diffusion coefficient is purely time dependent, and for later times the diffusion coefficient is proportional to $\varepsilon^{1 / 3} r^{4 / 3}$. A number of experiments support the existence of the two regimes. ${ }^{11-15,21}$

To date, the precise value of the Richardson constant $g$ is still not known. Experimental values for $g$ are difficult to obtain and have a considerable range. Tatarski ${ }^{35}$ reported 0.06 , a value that has to be regarded as an order-ofmagnitude estimate according to Fung and Vassilicos. ${ }^{8}$ Based on smoke plume experiments, $g=1.7$ was suggested by Byzova et al. ${ }^{36}$ Particle tracking velocimetry (PTV) experiments at $\operatorname{Re}_{\lambda} \approx 90$ and $\operatorname{Re}_{\lambda} \approx 170$ yielded $g \approx 0.5$ and $g$ $\approx 0.6$, respectively. ${ }^{11,21}$ For DNS simulations at $\operatorname{Re}_{\lambda}=200$ -283 , values of $g \approx 0.5-0.7$ are reported. ${ }^{12-14,21}$ The influence on dispersion dynamics of finite $\operatorname{Re}_{\lambda}$ flows is still subject of discussion. Exit time statistics introduced by Artale et al. $^{37}$ measure separation velocities at fixed scales. Employing the Richardson diffusion equation, $g \approx 0.5$ is found. In Bourgoin et al. ${ }^{15}$ it is inferred that in order to observe the Richardson-Obukhov law, a ratio of integral time to Batchelor time $\mathcal{T} / \tau_{\mathrm{B}}>10$ is required, with $\mathcal{T} / \tau_{\eta}=(\mathcal{L} / \eta)^{2 / 3}$, where $\mathcal{L}$ is the integral scale. They base this inequality on missing plateaus of the curves $\left(\left\langle r^{2 / 3}\right\rangle-r_{0}^{2 / 3}\right) /\left(r_{0}^{2 / 3} t / t_{0}\right)$ versus $t / \tau_{B}$. In the experiment reported by Berg et al., ${ }^{21}$ plateaus of the same curves were observed for $t>\tau_{B}$, despite the fact that $\mathcal{T} / \tau_{B}$ $<10$. In Ref. 21 , the relation

$$
\left\langle r^{2}(t)\right\rangle / r_{0}^{2}=g\left(t / \tau_{B}-T_{0} / \tau_{B}\right)^{3},
$$

introduced by Ott and Mann, ${ }^{11}$ is studied again. Provided that $\eta / r_{0} \sim 0$, this function describes well the particle separation, and it allows us to extract a Richardson constant $g$ from intermediate Reynolds number data. The function fits experimental and DNS data nicely. ${ }^{21}$ Throughout all these approaches, there seems to be a consensus that experiments in flows with a much more clearly developed inertial range are desirable.

From EDQNM, Novikov ${ }^{22}$ estimated $g=1.8$ and the analysis of Larcheveque and Lesieur ${ }^{23}$ yields $g=3.5$, while a revised application of the EDQNM approximation by Thomson $^{38}$ gives $g=1.4$. Kraichnan's LHDI ${ }^{18}$ also yields a diffusion equation for particle separation. However, probably due to the velocity field being $\delta$-correlated in time, the resulting Richardson coefficient is $g=5.5$, which is much higher than most current estimates. Further, it was shown ${ }^{11}$ that the predicted shape of the separation PDF is much less skewed and much less flat than was observed in a number of experiments. For a thorough discussion of the Kraichnan model, we refer again to Falkovich et al. ${ }^{20}$ Based on a differential equation for the mean square relative separation,
Franzese and Cassiani ${ }^{19}$ arrive at $g=0.64$ in the limit of high Reynolds number. Inertial subrange quantities such as the Lagrangian and Eulerian structure function Kolmogorov constants $C_{0}$ and $C_{k}$, respectively, and the Lagrangian velocity autocorrelation time $T_{\mathrm{L}}$ are an integral part of their derivation.

One approach to mimic turbulent flows with much higher Reynolds number is to use synthetic turbulence and specifically kinematic simulations (KS); e.g., Malik and Vassilicos. ${ }^{9}$ In kinematic simulation, the Eulerian velocity field $\mathbf{u}(\mathbf{x}, t)$ is approximated by a Fourier series with a finite number of modes. Modes are chosen to satisfy incompressibility, with spectral and spatial structure to be similar to real turbulence. The Fourier modes are uncorrelated and hence there is no "sweeping" of the smaller eddies by the larger eddies, just as in the Kraichnan model. Thomson ${ }^{39}$ argues that as a consequence and depending on the magnitude of the mean flow, $\left\langle r(t)^{2}\right\rangle$ can grow in proportion to $t^{9 / 2}$. The discussion is ongoing: Osborne et al. ${ }^{24}$ showed that while it is difficult to distinguish between regimes of $\left\langle r^{2}(t)\right\rangle \propto t^{3}$ and $\left\langle r^{2}(t)\right\rangle \propto t^{9 / 2}$, even at very high Reynolds number, KS clearly reproduces Richardson's diffusion prediction $d / d t\left\langle r^{2}(t)\right\rangle$ $\propto r^{4 / 3}$ over a wide range of scales. In addition, Ref. 24 points out that the major difference between the two results is the implementation of the integration time step. In $\mathrm{KS}$, the $\mathrm{Ri}$ chardson constant typically is $\mathcal{O}(0.01)$, and in Davila and Vassilicos $^{25}$ it is shown to be a decreasing function of the mean flow, which is, of course, not desirable. The KS approach, though, reproduces very well the evolution of flatness coefficients of the two-particle relative velocity components also for small initial particle separations $r_{0}$ and for different Reynolds numbers (e.g., Ref. 9).

In Ref. 25, the connection is made between Richardson pair diffusion and the fractal spatial distribution of straining stagnation points in the turbulent-like flow. The observation that "fluid element pairs follow close trajectories for long stretches of time and separate violently when they meet straining flow regions around stagnation points" 40 gave rise to the statistical persistence hypothesis (SPH). In Goto and Vassilicos, ${ }^{26}$ a corresponding pair diffusion model is developed with a PDF equation that is a generalization of Richardson's diffusion equation. The model predicts the Richardson constant as $g=6.9$.

Finally, we arrive at the class of Lagrangian stochastic models (LSMs) for pair diffusion. Durbin ${ }^{31}$ was one of the first to formulate a Lagrangian model for two-particle dispersion based on the Langevin equation for relative velocities. He reduced the dispersion problem in a real turbulent flow to a quasi-one-dimensional (1D) one. Thomson ${ }^{30}$ improved his model significantly by making it consistent with the "wellmixed condition"; i.e., an initially uniform particle distribution remains stationary in time. He found $0.9<g<3.4$. Variations were presented by Borgas and Sawford, ${ }^{41}$ Pedrizzetti and Novikov ${ }^{29}$ and Kurbanmuradov ${ }^{42} 0.8<g$ $<1.8$. Heppe ${ }^{28}$ typically gave smaller values $(0.1<g<0.4)$ depending on the choice of the Lagrangian structure function constant $C_{0}$.

The above stochastical dispersion models all assume that 
relative particle accelerations have negligible memory, which implies that Lagrangian position and velocity can be described by treating the latter as a Markov process. ${ }^{41}$ Heppe concluded from Yeung's ${ }^{43}$ results on relative acceleration that at small separations, which are governed by time scales of $\mathcal{O}\left(\tau_{\eta}\right)$, the Markov assumption is likely to fail. He expected qualitative deviations of a Markovian model from the dispersion physics. From DNS, Yeung ${ }^{43}$ found significantly long relative acceleration correlations, especially for small separations of $\mathcal{O}(\eta)$. While a few studies managed to measure acceleration variances ${ }^{33,34}$ and even acceleration auto-correlations ${ }^{32}$ at considerable Reynolds number, to our knowledge there are presently no experimental relative autocorrelation acceleration data available. However, there is no reason that the Markov approximation should not be valid in the inertial range. As was noted by Ref. 25, it is the omission of viscous, highly intermittent, non-Markovian effects that lead to an underestimation of, e.g., the flatness peak factor of particle distribution and relative velocities. ${ }^{28,29}$ In the latest Borgas and Yeung model, ${ }^{27}$ these viscous effects are taken into account. The drift term of the Langevin-type equation is derived from the Eulerian transport equation: relative acceleration is parametrized as a quadratic closure of relative velocity and a correction term that guarantees smooth transitions between the different regimes. The diffusion term is a scale-dependent Wiener process.

In principle, the 2004 Borgas and Yeung model could be used for our purpose. However, the explicit dependence on subtle Eulerian flow statistics such as $\partial \ln \left[P_{E}(u)\right] / \partial u$ and $\langle a ; u, r\rangle$ as well as the correction term has led us to derive and employ a much simpler stochastical model. Based on a K41-type scaling argument ${ }^{44}$ for the field of coarse grained velocity derivatives, we propose that a process similar to viscous material line stretching also occurs in the inertial range. In Naso and Pumir, ${ }^{45}$ evidence is given, based on DNS simulations at $\operatorname{Re}_{\lambda}=130$, that coarse grained strain $\widetilde{s}^{2}$ actually does scale according to $\mathrm{K} 41$ and that $\left\langle\widetilde{s}^{2}\right\rangle \sim r^{-4 / 3}$. Not just the magnitude but also the geometrical properties of velocity derivatives are reported to behave similarly in the inertial range as in the viscous range by Borue and Orzag ${ }^{46}$ and Tao et al. ${ }^{47}$ From DNS results and holographic particle image velocimetry (PIV) results, it is shown that a number of characteristic features of the velocity derivative tensor $A_{i j}$ $=\partial u_{i} / \partial x_{j}$ are also present in its coarse grained counterpart $\widetilde{A}_{i j}$. A detailed overview on these properties is given in Tsinober. ${ }^{48}$ Given these similarities, it is also natural to ask if the process of material line stretching has its counterpart in the inertial range. The main idea is that particle separation is governed by a time scale that increases with increasing particle distance $r(t)$. This captures the Lagrangian aspect of the process. In the viscous range the relevant time is $\tau_{\eta}$ and in the inertial regime, the separation dynamics become slower according to K41 scaling and the time scale should be $\propto \varepsilon^{-1 / 3} r^{2 / 3}$. Just like, e.g., Pedrizzetti and Novikov ${ }^{29}$ or Heppe, ${ }^{28}$ we thus also employ a diffusion coefficient that is dependent only on the separation and not on time. Such a diffusion coefficient was introduced by Richardson, ${ }^{1}$ and was inherently used by Obukhov ${ }^{16}$ and Batchelor ${ }^{49}$ to relate the
Kolmogorov spectrum $p=5 / 3$ to the $\left\langle r^{2}(t)\right\rangle=g \varepsilon t^{3}$ law. In the framework of KS, this locality assumption was revisited by Nicolleau and $\mathrm{Yu}^{50}$ and confirmed also for values of $p$ $\neq 5 / 3$. As will be shown below, a good estimate for this time scale is obtained from a parametrized form of the secondorder structure function $S_{2}(r) .{ }^{27}$ Linking the particle separation process to material line stretching, which is modeled as a Gaussian process with an evolving time scale, results in a relatively simple formulation of a quasi-1D stochastic model. The model has only one "free" model parameter for each of the viscous, inertial, and diffusive separation regimes.

Similar to the difficulties that arise due to finite Reynolds number, in addition, the influence of finite sized experimental observation domains has not yet been conclusively addressed. Mean values of $r^{2}(t)$ can be biased towards lower values if fast separating particle pairs have already left the observation domain at time $t$. It is thus desirable and in the scope of this work to study both the effect of finite Reynolds number and the effect of limited observation scale or time. The idea of this contribution is to reproduce known experimental dispersion data with a model as simple and physical as possible. The model is to be used to explore the influence of initial particle separation $r_{0}$, the influence of various observation domain scales, and the influence of various ratios of $\mathcal{L} / \eta$; e.g., to mimic the dispersion process at various Reynolds numbers. Essentially, the proposed model is meant to check how far the analogy to viscous material line stretching can be exploited for two-particle separation. In addition, it is meant to be a simple design tool for experiments that will allow more insight into the particle dispersion process.

The paper is organized as follows. In the next section, a very brief overview on material line stretching is given. In the modeling section expressions are derived to mimic the process of particle dispersion. We outline how finite observation domains are simulated. In Sec. III we show how the model compares with data from existing experiments. In Sec. IV the model is used to explore the influences of finite field of view and of the scale separation $\mathcal{L} / \eta$. In Sec. V we make a summary and draw some conclusions.

\section{MATERIAL LINE STRETCHING}

The process of infinitesimal material line stretching has been studied extensively ${ }^{48,51-55}$ and is described by the kinematic relation

$$
\frac{1}{2} \frac{d r^{2}}{d t}=r_{i} r_{j} s_{i j}
$$

with $\mathbf{r}=\mathbf{x}^{(2)}-\mathbf{x}^{(1)}$ denoting the particle separation and $s_{i j}$ $=\frac{1}{2}\left(\partial u_{i} / \partial x_{j}+\partial u_{j} / \partial x_{i}\right)$ the rate of strain tensor. The stretching rate has been measured, both in direct numerical simulations and with particle tracking velocimetry. ${ }^{14,21,51,53-55}$ It has been found that the nondimensionalized stretching rate

$$
L(t)=r_{i}(t) r_{j}(t) s_{i j}(t) \frac{\tau_{\eta}}{r^{2}},
$$

with the Kolmogorov time scale, $\tau_{\eta}=(\nu / \varepsilon)^{1 / 2}$, is seemingly independent of $\operatorname{Re}_{\lambda}$, at least for $\operatorname{Re}_{\lambda}<300$. After a time of 
$t / \tau_{\eta} \approx \mathcal{O}(1)$, the stretching rate takes on a mean value in the range of $\langle L(t)\rangle \approx 0.11-0.14$, which is equal to about $0.4\left\langle\Lambda_{1}(t)\right\rangle \tau_{\eta} . \Lambda_{1}(t)$ is the largest principal eigenvector of $s_{i j}(t)$. It is not clear how the slight discrepancy between the different measurements can be explained. For both experimental ${ }^{21,54,55}$ and $\mathrm{DNS}^{14,51}$ investigations, the same range of stretching rates has been found, with no systematic dependence; e.g., on Reynolds number.

$\langle L(t)\rangle$ can be understood better if interpreted geometrically by decomposing Eq. (3) into its terms that are associated with each eigenvalue $\Lambda_{i}$ and eigenvector $\boldsymbol{\lambda}_{i}$ of the rate of strain tensor $s_{i j}{ }^{48}$ as

$$
L(t)=\tau_{\eta} \sum_{i} \Lambda_{i}(t) \cos ^{2}\left[\boldsymbol{\lambda}_{i}(t), \mathbf{r}(t)\right] .
$$

From (4) it becomes clear that $L(t)$ depends on the alignment to the principal strain axis of $s_{i j}(t)$ as well as on the magnitude of the associated eigenvalues. Batchelor's assumption ${ }^{56}$ of full alignment of $\mathbf{r}(\mathbf{t})$ with $\boldsymbol{\lambda}_{1}(t)$ was corrected by Girimaji and Pope, ${ }^{51}$ who showed that, due mainly to rotation of the strain eigenframe, only about $40 \%$ of the maximal theoretical stretching actually occurs (see also Dresselhaus and Tabor ${ }^{52}$ and Guala et $\mathrm{ll}^{55}$ ). For the mean of Eq. (4), an upper value of $\langle L(t)\rangle \leqslant 0.5$ can be derived as follows. If we use the convention $\Lambda_{1}>\Lambda_{2}>\Lambda_{3}, \Lambda_{1}+\Lambda_{2}+\Lambda_{3}=0$, due to incompressibility and the fact $\left\langle\Lambda_{2}(t)\right\rangle>0$, we can express $\left\langle\Lambda_{1}(t)\right\rangle$ in terms of $s^{2}=s_{i j} s_{i j}$ as

$$
\left\langle\Lambda_{1}(t)\right\rangle<\frac{\left\langle\sqrt{s^{2}}\right\rangle}{\sqrt{2}} .
$$

For the Kolmogorov time, we have an upper bound as

$$
\tau_{\eta}=\frac{1}{\sqrt{2\left\langle s^{2}\right\rangle}}<\frac{1}{\sqrt{2}\left\langle\sqrt{\left.s^{2}\right\rangle}\right.} .
$$

Assuming full alignment of $\mathbf{r}(\mathbf{t})$ with $\boldsymbol{\lambda}_{1}(t)$ and using (5) and (6), an upper bound for the average of (4) can be written as

$$
\langle L(t)\rangle\left\langle\frac{1}{\sqrt{2}\left\langle\sqrt{s^{2}}\right\rangle} \frac{\left\langle\sqrt{s^{2}}\right\rangle}{\sqrt{2}}=\frac{1}{2} .\right.
$$

A nonperfect alignment of $\mathbf{r}(t)$ with $\boldsymbol{\lambda}_{1}(t)$, a positive intermediate eigenvalue $\left\langle\Lambda_{2}(t)\right\rangle>0$, and a flat distribution of $s^{2}$ will all contribute to reduce the actual mean value. From, e.g., the PTV data of $\operatorname{Re}_{\lambda} \approx 70$ turbulence, ${ }^{21}$ we measure $\left\langle\cos ^{2}\left[\mathbf{r}(t), \boldsymbol{\lambda}_{1}(t)\right]\right\rangle \approx 0.35, \quad\left\langle\Lambda_{1}\right\rangle=0.9\left\langle\sqrt{s^{2}}\right\rangle / \sqrt{2}, \quad$ and $\quad \tau_{\eta}$ $=0.9 /\left(\sqrt{2}\left\langle\sqrt{s^{2}}\right\rangle\right)$, which is consistent with a mean stretching rate of $\langle L(t)\rangle \approx 0.14$ that is also reported in Berg et al. ${ }^{21}$ The PDFs of $L(t)$ for different times and initially randomly oriented $\mathbf{r}(t)$ is shown in Fig. 1.

\section{MODELING}

The stochastical model that we present here is quasi-1D with Langevin-type equations for the evolution of relative particle separation. From Fig. 1(a) we observe that the complex underlying processes lead to a relatively simple overall behavior of the nondimensional stretching rate $L_{\eta}(t)$. The strain tensor evolves in time with changing intensity, shape, and orientation of its principal strain frame. Infinitesimal ma-

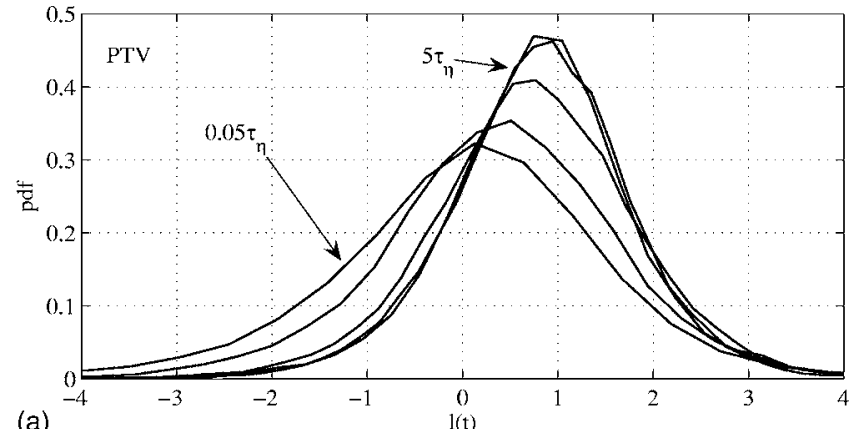

(a)

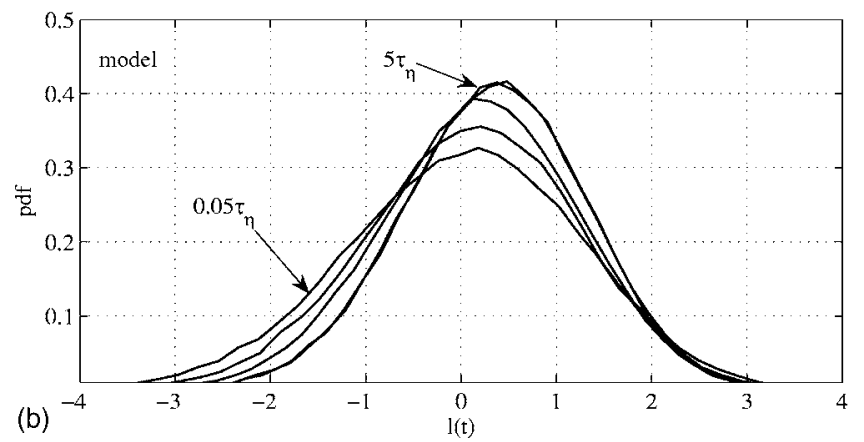

FIG. 1. (a) PDFs of the measured stretching rate $l(t)=L(t)$ for different times, $t / \tau_{\eta}=0.05,0.5,1.5,3,5$, from bottom to top. The measurements are from the particle tracking experiment (PTV), described in Ref. 21. (b) PDFs of the modeled $l(t)$ for different times, $t / \tau_{\eta}=0.05,0.5,1.5,3,5$, from bottom to top.

terial elements experience nonpersistent straining because of these dynamics. ${ }^{51,52,55}$ The overall stretching rate for $r \ll \eta$ we find, however, to a first approximation normally distributed around an asymptotic mean value $\bar{L}_{\eta}$. The asymptotic state is reached after a time $\alpha_{\eta} \tau_{\eta}$, where $\alpha_{\eta} \sim \mathcal{O}(1)$. As will become clearer below, it is convenient to express this time in terms of the Kolmogorov frequency $f_{\eta}=1 / \tau_{\eta}$ as $\alpha_{\eta} / f_{\eta}$. Close inspection of measured $L_{\eta}(t)$ data reveals that the distribution is slightly skewed and that the flatness coefficient is slightly above Gaussian. In order to keep the model as simple as possible, these properties will, however, be neglected in our modeling. Further, we assume that $d L_{\eta}(t) / d t$ has a negligible memory in comparison to $L_{\eta}(t)$ itself. Keeping in mind our introductory discussion on the implications of such a Markov approximation, we try to show below [Fig. 1(b)] that in fact the evolution of $L(t)$ can then be simulated reasonably well with a 1D Ornstein-Uhlenbeck process in the viscous regime. Below, when we show results for scales $r \gg \eta$, we will find that the model performance improves further, from fair to good. This reflects what was already noted by other authors: ${ }^{14,27,28}$ the omitted viscous effect leading to enhanced intermittency acts mainly on small scales. The prize for the simplification that allows us to use a 1D Ornstein-Uhlenbeck process has to be paid mainly at small separations.

For the viscous regime $r \ll \eta$, we have

$$
d L_{\eta}(t)=-\frac{f_{\eta}}{\alpha_{\eta}}\left[L_{\eta}(t)-\bar{L}_{\eta}\right] d t+\sigma \sqrt{\frac{2 f_{\eta}}{\alpha_{\eta}}} d W(t) .
$$

$d W(t)$ is a Wiener process with the properties $\langle d W(t)\rangle=0$ and $\left\langle d W(t) d W\left(t^{\prime}\right)\right\rangle=\delta\left(t-t^{\prime}\right) d t$ for $\left(t \neq t^{\prime}\right)$. Throughout the model, 
$f$ has unit $\left[\mathrm{s}^{-1}\right], t$ has $[\mathrm{s}], W$ has $\left[\mathrm{s}^{1 / 2}\right], r$ has [m]; all other variables and parameters are dimensionless. The normally distributed $L_{\eta}(t)$ has an auto-correlation time $\alpha_{\eta} \tau_{\eta}$ and a variance $\sigma^{2}$. With (2) and (3) we can write

$$
d r(t)=r(t) f_{\eta} L_{\eta}(t) d t .
$$

Expressions (8) and (9) depend on the "free" parameters $\alpha_{\eta}$, $\bar{L}_{\eta}$, and $\sigma$. We will now expand the range of validity for (9) to larger separations up to $r>\mathcal{L}$ by letting the time scale $\alpha_{\eta} / f_{\eta}$ become a function of the separation as $\alpha[r(t)] / f[r(t)]$. This represents the key idea of the presented model. This essentially makes the process a Lagrangian one, and it is in this step that we employ the assumption of a diffusion coefficient depending only on separation. With the help of the second-order structure function, we define a time scale that is a function of the separation $r(t)$.

In addition, by dividing (8) with $\sigma$ and by employing the well-mixed condition, the number of free parameters will ultimately be reduced from 3 to just 1 for each of the viscous, inertial, and diffusion regimes. With (8) and (9), the longitudinal second-order structure function can be expressed for the viscous regime as

$$
S_{2}^{\eta}(r)=\left\langle\left(\frac{d r}{d t}\right)^{2}\right\rangle=r(t)^{2} f_{\eta}^{2} \sigma^{2} .
$$

From (10), it follows that a frequency $f(r)$ that substitutes $f_{\eta} \sigma$ can be expressed in terms of $S_{2}(r)$ not just for the viscous regime but for all $r(t)$ as

$$
f(r)=\sqrt{S_{2}(r) / r(t)^{2}} .
$$

If we assume that the dispersion process occurs in a homogeneous and isotropic flow, the integral scale $\mathcal{L}$ and the rate of dissipation $\varepsilon$ are sufficient to describe the flow properties. For this case it is convenient to use the parametrized form of $S_{2}(r)$ employed by Borgas and Yeung ${ }^{27}$

$$
\begin{aligned}
S_{2}(r)= & 2\left[1-\exp \left(-\frac{r}{\left(15 C_{k}\right)^{3 / 4} \eta}\right)\right]^{4 / 3} \\
& \times(\varepsilon \mathcal{L})^{2 / 3}\left(\frac{r^{4}}{\frac{64 \mathcal{L}^{4}}{C_{k}^{6}}+r^{4}}\right)^{1 / 6},
\end{aligned}
$$

with the viscosity $\nu$ and the Kolmogorov constant $C_{k}$. For $C_{k}$ we use the approximation

$$
\begin{aligned}
& C_{0}=\frac{7.0}{1+(7.5)\left(7.0^{2}\right) \mathrm{Re}_{\lambda}^{-1.64},} \\
& C_{k}=\frac{2.1}{\left(1+7.5 C_{0}^{2} \operatorname{Re}_{\lambda}^{-1.64}\right)^{2 / 3}},
\end{aligned}
$$

as proposed by Sawford. ${ }^{57}$ For $\operatorname{Re}_{\lambda}$ we use

$$
\operatorname{Re}_{\lambda}=\sqrt{15}\left(\frac{\mathcal{L}}{\eta}\right)^{2 / 3}
$$

Adjusting (8) and (9) to $f_{\eta} \rightarrow f(r)$, we substitute $\alpha_{\eta} \sigma$ with $\alpha(r)$. Further, expression (8) is divided by $\sigma$, and with $l(t)$ $=L(t) / \sigma$, we get the final form of the model as

$$
d l(t)=-\frac{f(r)}{\alpha(r)}[l(t)-\bar{l}(r)] d t+\sqrt{\frac{2 f(r)}{\alpha(r)}} d W(t)
$$

and

$$
d r(t)=r(t) f(r) l(t) d t .
$$

The number of "free functions" is thus reduced to $2: \bar{l}(r)$ and $\alpha(r)$. Through $\alpha(r) / f(r)$, a time scale is now defined for all ranges and for $\eta \ll r \ll \mathcal{L}$, we have $\alpha(r) / f(r) \propto r^{2 / 3}$, like Ref. 1,28 , and 29, and consistent with K41 arguments. The phenomenological interpretation of this is that coarse grained material lines, in our case, the separation vectors $\mathbf{r}$, are stretched by a coarse grained strain field.

For these stochastic differential equations, we can write the corresponding Fokker-Planck equation ${ }^{58}$ as

$$
\begin{aligned}
\frac{\partial P}{\partial t} & +\frac{\partial}{\partial r} f(r) r(t) l(t) P-\frac{f(r)}{\alpha(r)} \frac{\partial}{\partial l}[l(t)-\bar{l}(r)] P \\
& -\frac{f(r)}{\alpha(r)} \frac{\partial^{2}}{\partial l^{2}} P=0 .
\end{aligned}
$$

To ensure that the well-mixed condition ${ }^{30}$ is fulfilled, the probability distribution $P(r, t)$ needs to be stationary in time; i.e., an initially uniformly distributed set of particle separations has to remain uniformly distributed for all times. A solution of the form $P=r^{2} e^{-l^{2} / 2}$ exists provided that

$$
\frac{\bar{l}(r)}{\alpha(r)}=\frac{\partial}{\partial r}\left[f(r) r^{3}\right] \frac{1}{f(r) r^{2}} .
$$

With the additional condition of (18), the number of free functions is thus reduced to 1 ; i.e., it is enough to define $\bar{l}(r)$. As can be seen from Fig. 2, there are three distinct regimes arising from (12): a viscous regime, the inertial regime itself, and a diffusive regime. We expect the separation processes to be similar but not identical in the three different regimes. In the viscous regime, separation is driven by only the viscous scale. In the inertial range, however, the scale associated with $r(t)$ is governing, but it is not alone. In the diffusive regime, the dominant time scale is essentially frozen to the value associated with $\mathcal{L}^{2 / 3}$, and therefore we can expect $\bar{l}(r)$ to again change. We thus create a function $\bar{l}(r)$ with three discrete levels: $\bar{l}_{\nu}, \bar{l}_{i}$, and $\bar{l}_{d}$, and let the transitions between them be governed by the properties of (18) as

$$
\bar{l}(r)=\bar{l}_{d}+\mathcal{L}^{4} \frac{e^{-r /\left(15 C_{k}\right)^{3 / 4}} \eta\left[\bar{l}_{\nu}+l_{i}\left(e^{r /\left(15 C_{k}\right)^{3 / 4} \eta}-1\right)\right]-\bar{l}_{d}}{\mathcal{L}^{4}+C_{k}^{6} r^{4} / 64} .
$$

The function (19) is plotted in Fig. 3 for $\bar{l}_{\nu}=0.39, \bar{l}_{i}=0.71$, and $\bar{l}_{d}=0.34$. These three values are obtained from fitting the model to all the available experimental and numerical data. They are used unchanged for all simulations that are presented in the following. It is difficult to measure and thus to check the above model parameters by experiment. It is required to measure $\bar{l}(r)$ for pairs that have started their evolution at initial separations $r_{0} \ll r$, and the pairs need to be observed for a long time $t_{o}$, so that $r \ll \bar{r}\left(t_{o}\right)$. Otherwise, the 


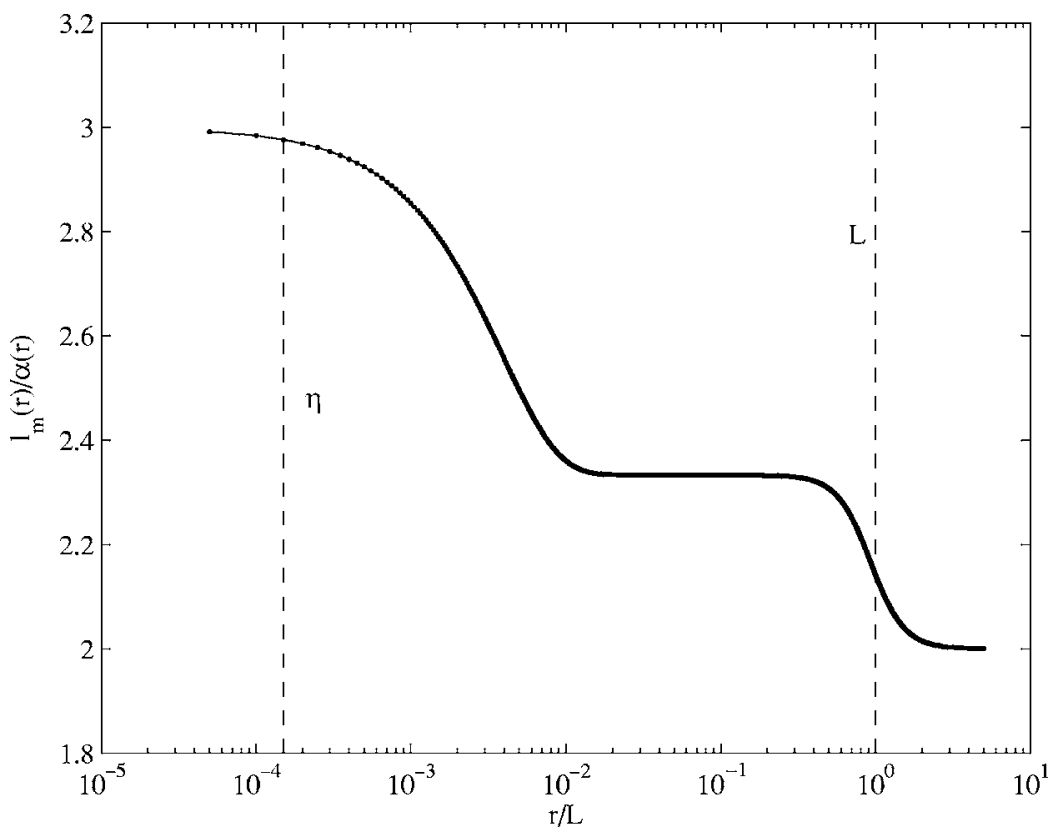

FIG. 2. The ratio of $\bar{l}(r) / \alpha(r)$ that is required in order for the well-mixed condition to be fulfilled is plotted vs $r / \mathcal{L}$.

statistics of $r(t)$ are either contaminated by $r(t)$ that are "younger" than $\alpha(r) / f(r)$, or if the observation time is to short, only "fast" $r(t)$ contribute to $\bar{l}(r)$. From an analysis of DNS data presented in Biferale et al., ${ }^{14}$ it is possible, though, to get an estimate for $\bar{l}_{i}$, the parameter for the inertial range. For a set of $r$ with initial separation $r_{0} / \eta=1.2$, we obtain $\bar{l}_{i}$ $\approx 0.75$ at $50<r / \eta<100$. Additional support for the chosen values can be gained via a comparison with the drift term characteristic of the model proposed by Borgas and Yeung. ${ }^{27}$ Their drift term [Fig. 4 of Ref. 27] behaves neutrally for $u_{\|} / \sigma \approx 0.25$ for viscous separations and for $u_{\|} / \sigma \approx 0.7$ for inertial separations, which is close to our values.

Two randomly selected particles are initially not predominately aligned with the principal strain axis. The asymptotic state of mean alignment of $\mathbf{r}(t)$ with $\lambda_{1}(t)$ is reached only after a characteristic time $\alpha(r) / f(r)$. This alignment effect is modeled with the initial condition $\langle l(0, r)\rangle=0$. The such modeled viscous $l(t)$ behaves similarly but not identical to the measurements and is shown in Fig. 1(b). The initial separations used for this figure are very small, and thus during the course of their evolution do not exceed the viscous range; i.e., $r \ll \eta$. The lower value for the modeled $\bar{l}_{\nu}$, as compared to the mean shown in Fig. 1(a) obtained from PTV experiment, is reflecting the missing intermittency effects in the model, as was discussed in Sec. I. In reality, the distribution of $l(r)$ is somewhat flatter and more skewed around a higher mean value.

Recently, the role of backwards dispersion has been emphasized by Sawford ${ }^{59,60}$ and experimental evidence of the phenomenon has been reported in Berg et al. ${ }^{21}$ As already

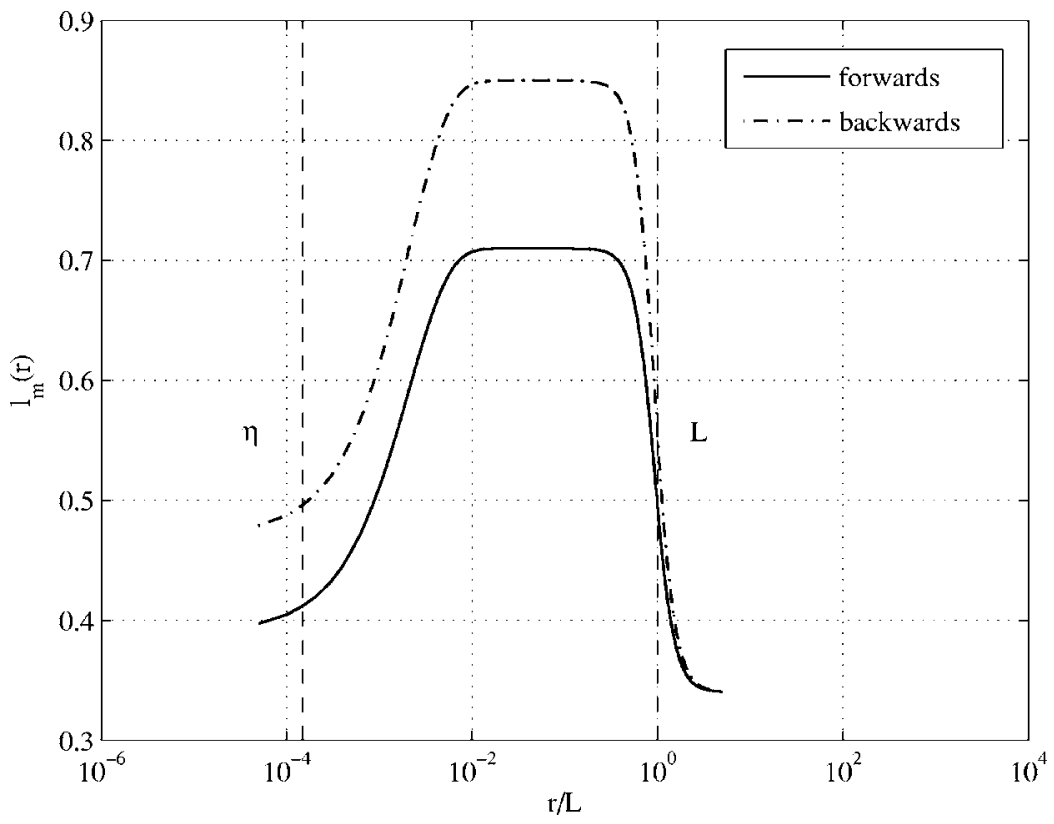

FIG. 3. $\bar{l}(r)$ as obtained from (19) is plotted vs $r / \mathcal{L}$ for forwards (solid line) and backwards (dash-dot line) case. 
mentioned in Ref. 21, a characteristic feature of particle separation with time going backwards is that material line elements tend to align with the most compressing eigenvector of the rate of strain tensor. Despite the fact that this was observed and reported only for the viscous range, there are good reasons to assume and also preliminary experimental evidence to believe that the same is true also in the inertial range. Due to $\left\langle\Lambda_{2}(t)\right\rangle>0$ in turbulent flows and due to incompressibility, i.e., $\Lambda_{i}(t)=0$, we have that $\left\langle\left|\Lambda_{3}(t)\right|\right\rangle$ $>\left\langle\Lambda_{1}(t)\right\rangle$. Consequently, the mean stretching rate backwards in time should be higher than the mean stretching rate forwards in time. From PTV experiments, ${ }^{21}$ a ratio of $\langle L(t)\rangle_{\text {back. }} \approx 1.25\langle L(t)\rangle_{\text {forw. }}$ was measured in the viscous range. The parameters $\bar{l}_{\nu}$ and $\bar{l}_{i}$ are thus multiplied with this factor of 1.25 to model the case of backwards dispersion. Beyond the inertial range odd moments vanish and $\left\langle\Lambda_{2}(t)\right\rangle$ $=0$, if coarse grained at $r>\mathcal{L}$, and thus $\bar{l}_{d}$ can be left unchanged. Expression (19) is computed for the values $\bar{l}_{\nu}$ $=0.49, \bar{l}_{i}=0.89$, and $\bar{l}_{d}=0.34$, and the resulting $\bar{l}(r)$ is plotted in Fig. 3 denoted as a dash-dotted line. From Eq. (18), it follows that with an increased value for $\bar{l}(r), \alpha(r)$ should also increase. This is fully consistent with Sawford, ${ }^{60}$ who interprets the Lagrangian time scale as the memory effect of the flow and finds that the case of backwards dispersion is associated with a longer memory; i.e., a larger time scale.

With the model introduced and its parameters defined, it can be run for any scale separation $\mathcal{L} / \eta$ for any initial separation $r_{0}$. However, in order to also compare the model with experimental results, one important feature is still missing: Real experiments always have only a finite field of view, resulting in a finite observation scale $d$. A finite field of view causes particles with a separation much larger than their corresponding mean to leave the field of view "too early," which will lead to a truncation of the separation distribution. Sweeping velocities that translate separating particle pairs across the field of view make the situation even worse. In the following, we explain how this effect is captured in the presented model.

The principal idea is to use Sawford's stochastic oneparticle model ${ }^{57}$ for the evolution of the center of mass of evolving particle pairs. Each moving center of mass is started at a random position inside a virtual observation domain. Together with the modeled evolving relative separation, the particle positions of the pairs are defined. The orientation of the pair is chosen randomly for each realization and is fixed in time. As soon as the first of the particles leaves the virtual domain, the separating pair is considered "lost" for the statistics.

The stochastic model is a Langevin equation describing a particle's Lagrangian acceleration $A(t)$ in an asymptotically stationary process as

$$
d A(t)+\alpha_{1} A(t) d t+\alpha_{2} \int_{0}^{t} A\left(t^{\prime}\right) d t^{\prime} d t=\sqrt{2 \alpha_{1} \alpha_{2} \sigma_{m}^{2}} d W(t)
$$

with

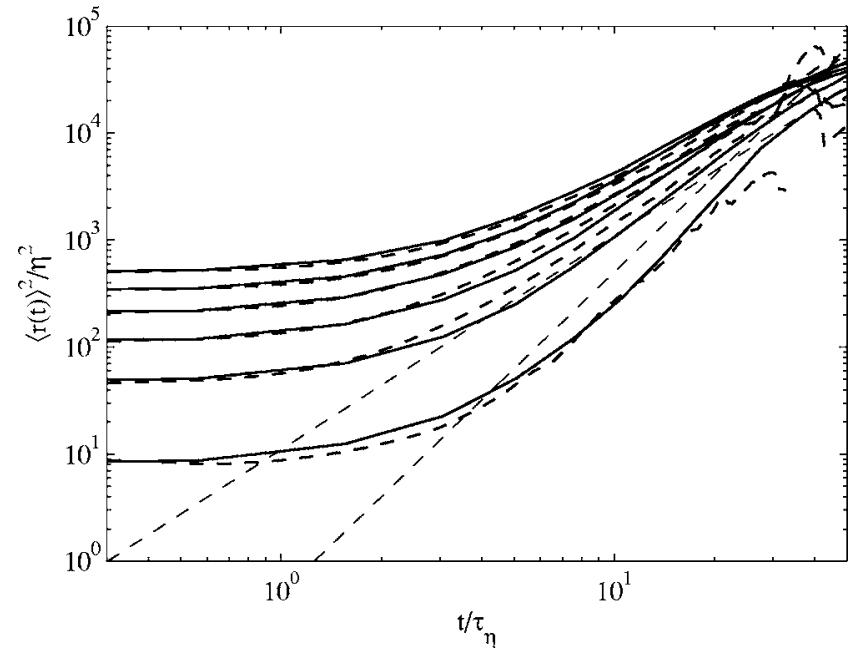

FIG. 4. Comparison of particle separations as obtained from particle tracking (dashed lines) versus model prediction (solid lines). Initial separations $r_{0} / \eta=3,7,11,14,18$. The straight dashed lines are $y \propto t^{2}$ and $y=0.5 \varepsilon t^{3}$.

$$
\begin{aligned}
& \alpha_{1}=-\left(\beta_{1}+\beta_{2}\right), \\
& \alpha_{2}=\beta_{1} \beta_{2}, \\
& \beta_{1}=C_{0} \varepsilon /\left(2 \sigma_{m}^{2}\right), \\
& \beta_{2}=-\left(2 a_{0} / C_{0}\right) \sqrt{\varepsilon / \nu}, \\
& a_{0}=5 /\left(1+110 / \operatorname{Re}_{\lambda}\right), \\
& \sigma_{m}^{2}=\frac{1}{2}\left[\sigma_{p}^{2}+R_{u}(r) \sigma_{p}^{2}\right] \\
& \sigma_{p}=\sqrt{\operatorname{Re}_{\lambda}}(\varepsilon \nu / 15)^{1 / 4}, \\
& R_{u}(r)=-\left(S_{2}-2 \sigma_{p}^{2}\right) /\left(2 \sigma_{p}^{2}\right) .
\end{aligned}
$$

The only difference to Sawford ${ }^{57}$ is that the velocity variance of the center of mass drops to half the value of a single particle, i.e., $\sigma_{m}^{2}=\frac{1}{2} \sigma_{p}^{2}$, when the velocities of the two particles decorrelate.

\section{RESULTS}

\section{A. Particle tracking and model, $\mathbf{R e}_{\lambda}=\mathbf{1 7 0}$}

All results that are presented in the following are obtained from Monte Carlo simulations of the above-described model with 10000 realizations for each of the different conditions. As a first result we show in Fig. 4 how the model predictions compare to measure data obtained from a particle tracking velocimetry (PTV) experiment at $\operatorname{Re}_{\lambda} \approx 170 .{ }^{21}$ For the PTV experiment, an integral scale of $\mathcal{L}=48 \mathrm{~mm}$ and a Kolmogorov length of $\eta=0.25 \mathrm{~mm}$ are measured so that $\mathcal{L} / \eta=190$. The observation domain $d$ is $150 \mathrm{~mm}$. In Fig. 4, where $\left\langle r^{2}\right\rangle$ is plotted over time for different initial separations, we see that there is good agreement between measured and modeled data. 


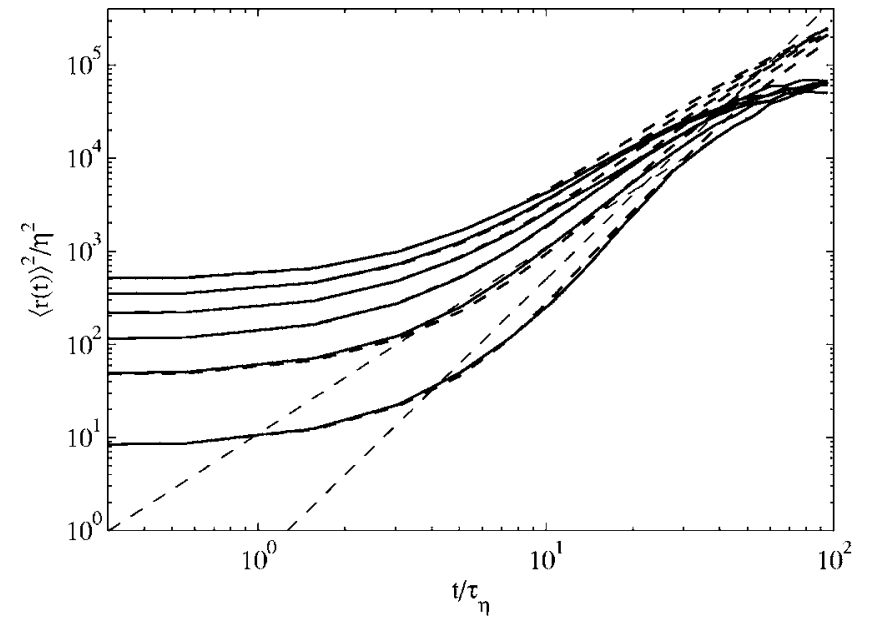

FIG. 5. Comparison of mean square separations as obtained with $d$ $=150 \mathrm{~mm}$ (solid lines) and $d=\infty$ (dashed lines). Initial separations $r_{0} / \eta$ $=3,7,11,14,18$. The straight dashed lines are $y \propto t^{2}$ and $y=0.5 \varepsilon t^{3}$.

We do not observe the Richardson-Obukhov law, and only the runs for the two smallest initial separation assume something like a $t^{3}$ slope.

To see to what degree the finite field of view $(d$ $=150 \mathrm{~mm}$ and $d / \mathcal{L} \approx 3$ ) influences these results, we show in Fig. 5 the evolution of the mean square separation with and without a finite field of view. After about $t / \tau_{\eta}=40 \approx \mathcal{T}$, the separations for the two cases become different. At this late time the separations are already $\mathcal{O}(\mathcal{L})$ and the finite observation scale effect is thus negligible, i.e., the reason that no Richardson-Obukhov law is observed clearly is the too low Reynolds number.

\section{B. DNS and model, $R e_{\lambda}=280$}

At slightly higher Reynolds number, i.e., $\operatorname{Re}_{\lambda}=280$, we simulate with $\mathcal{L} / \eta=628$ and $d=\infty$ the conditions of the DNS experiment reported by Biferale et al. ${ }^{14}$ In Fig. 6 mean square separation is plotted over time for different initial

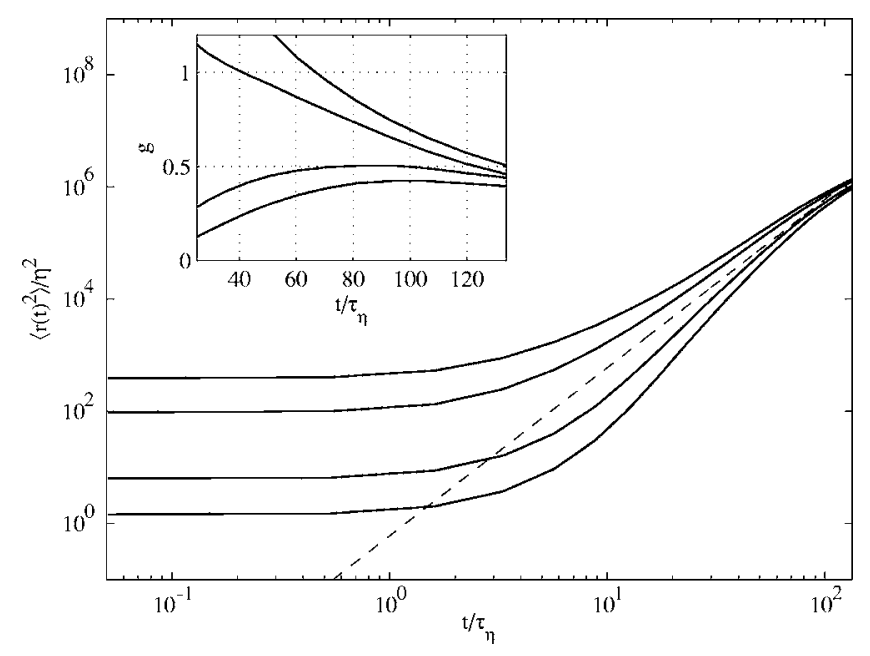

FIG. 6. Model predictions for particle separations over time and $g$ $=\left\langle r(t)^{2}\right\rangle /\left(\varepsilon t^{3}\right)$ (inset), for conditions as in Ref. 14. Initial separations $r_{0} / \eta$ $=1.2,2.5,10,20$. The dashed line is $y=0.6 \varepsilon t^{3}$.

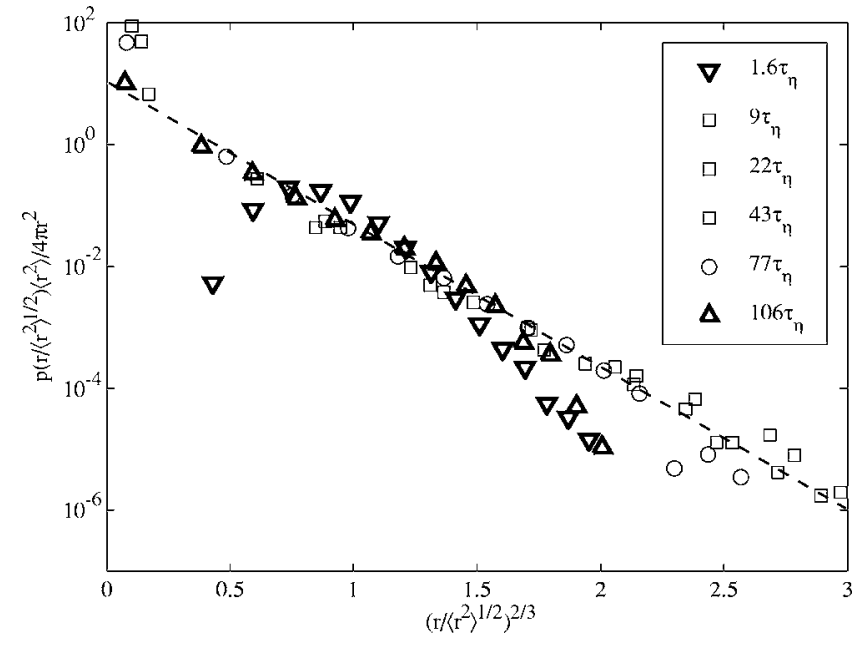

FIG. 7. Model predictions for the distance neighbor functions at different times, for $r_{0} / \eta=1.2$, and for conditions as in Ref. 14. Straight dashed line denotes the solution as obtained from the Richardson diffusion equation.

separations, $r_{0} / \eta=1.2,2.5,10,20$, as in the DNS case. In the inset, the Richardson constant as obtained from $g$ $=\left\langle r^{2}\right\rangle /\left(\varepsilon t^{3}\right)$ is plotted as a function of time. From both figures we observe that at around $t / \tau_{\eta} \approx 80$, the particle separation dynamics become less intense. This is reflected in a slight departure from the $t^{3}$ slope for $\left\langle r^{2}\right\rangle$ and in the maximum of the values for $g$ for the two small initial separations, shown in the inset. The separations start to feel the influence of the finite inertial range. In this sense, there is again good agreement between modeled and measured data.

The effect of the finite inertial range is shown more clearly in Fig. 7, where distant neighbor functions for $r_{0} / \eta$ $=1.2$ for various times are plotted. For times $9 \leqslant t / \tau_{\eta} \leqslant 77$, they all collapse around the distribution, as predicted by the Richardson diffusion equation. For very early times and for times $t / \tau_{\eta} \geqslant 80$, the distributions are closer to Gaussian.

We also measure the correlation of the relative separation velocity along the particle pair trajectories. In the inset of Fig. 8 we plot $D(t, \tau)=\left\langle\delta u_{\|}(t) \delta u_{\|}(t+\tau)\right\rangle$ versus $t / \tau_{\eta}$ for pairs with initial separation $r_{0}=1.2 \eta$. As already reported, ${ }^{14}$ $D(t, \tau)$ broadens with increasing travel time, confirming that the separation velocities decorrelate more slowly at larger travel times. In the body of the same figure we plot the same data rescaled with the time $t_{0}$ at which $D(t, \tau)=0$. The good agreement with Fig. 12 of Ref. 14 validates that the characteristic time-prefactor $\alpha(r)$, as obtained from Eq, (18), is consistent not only with the well-mixed condition, but also with actual turbulent flows, as obtained from DNS results.

Model results for forwards and backwards dispersion mimicking the case of $\operatorname{Re}_{\lambda}=280$ DNS conditions $(\mathcal{L} / \eta$ $=628$ and $d=\infty)$ are presented in Fig. 9. Particle separations $\left\langle r^{2}(t)\right\rangle$ are plotted over time $t-T_{0}$, as proposed and employed by Ott and Mann ${ }^{11}$ and recently by Berg et al. ${ }^{21}$ Basically, $T_{0}$ accounts for the processes occurring in the initial phase of separation; in our frame of reference this would be the alignment of $\mathbf{r}(t)$ with the principal strain axis of the corresponding scale. Not surprisingly, $T_{0}$ is always found to be close to $\tau_{\mathrm{B}}{ }^{21}$ The two dashed lines are $y=0.6 \varepsilon t^{3}$ and $y=1.2 \varepsilon t^{3}$; the 


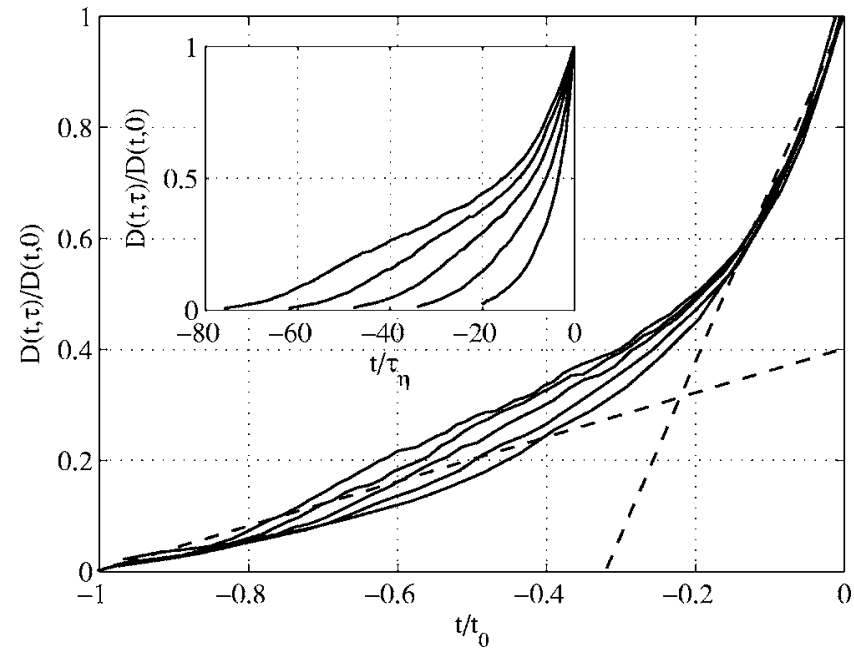

FIG. 8. Model predictions for the normalized correlation function $D(t, \tau) / D(t, 0)$ vs $t / t_{0}$, with $t_{0}$ as the time where the correlation crosses zero, as shown in the inset. Conditions are as in Ref. $14 ; r_{0} / \eta=1.2$. Straight dashed lines denote results reported in Ref. 14 .

ratio of the Richardson constants for backwards and forwards dispersion is thus found to be $g_{b} / g_{f} \approx 2$. This result is in good agreement with Ref. 21, where also $g_{b} / g_{f} \approx 2$ is reported. The ratio is clearly lower than the values $g_{b} / g_{f}$ $\approx 5-20$ proposed in Ref. 59 .

The modeled dispersion is consistent also with exit time statistics introduced by Artale et al. ${ }^{37}$ We show in Figs. 10 and 11 results for exit times, for Lyapunov exponents, and for Richardson constants, as employed in Ref. 14. $T(r)$ is defined as the average time it takes for a separation $r$ to reach the sphere $r_{n+1}$ from the sphere $r_{n}$, with $r_{n}=\rho^{n} r_{0}, n$ $=1,2,3 \ldots$, and $\rho=1.25$. The choice of $\rho=1.25$ is common but arbitrary, however, the sensitivity of the results to $\rho$ is very low. Following Ref. 14, the Lyapunov exponent and the Richardson constant can be obtained from $T(r)$ as

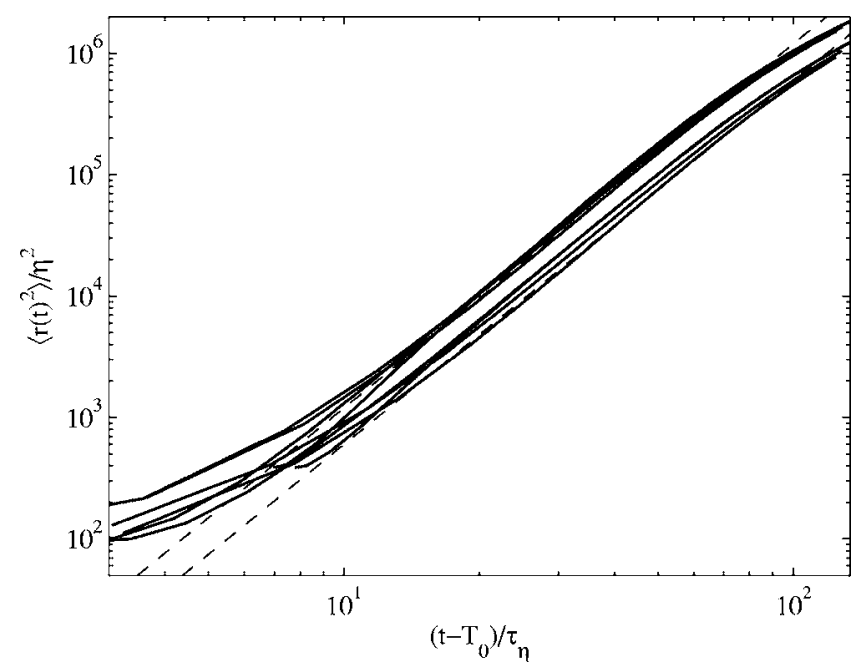

FIG. 9. Model predictions for the forwards and backwards dispersion. Conditions are as in Ref. 14; initial separations $r_{0} / \eta=1.2,2.5,10,20$. The dashed lines are $y=0.6 \varepsilon t^{3}$ and $y=1.2 \varepsilon t^{3}$.

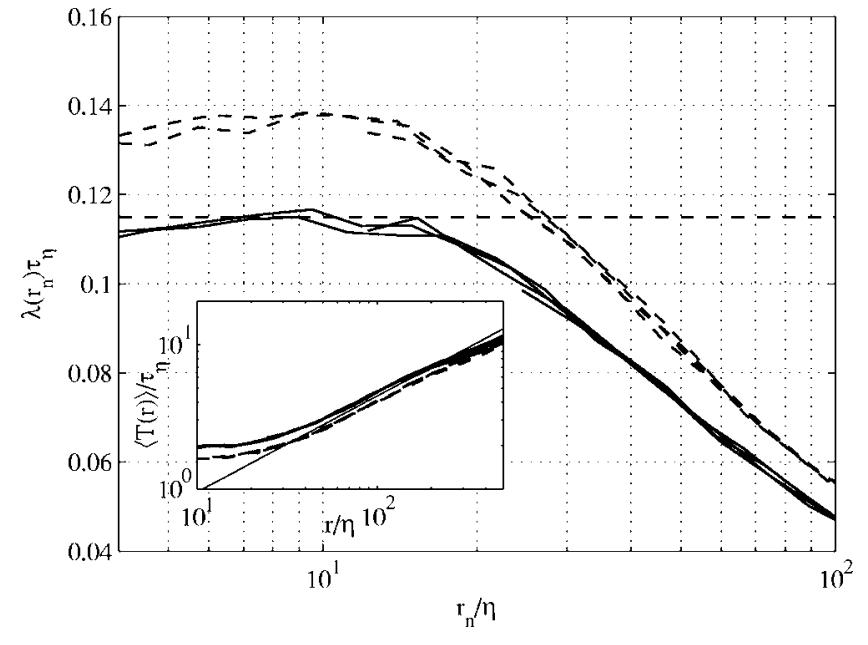

FIG. 10. Model predictions for the finite sized Lyapunov exponents for forwards and backwards dispersion with $\rho=1.25$; dashed lines are for the backwards case, $\operatorname{Re}_{\lambda}=280$; initial separations $r_{0} / \eta=1.2,2.5,10,20$. The straight dashed line denotes the result obtained by Biferale et al. (Ref. 14); $\lambda\left(r_{n}\right) \tau_{\eta}=0.115$. Inset: Model predictions for mean exit times, dashed lines are for the backwards case: $\operatorname{Re}_{\lambda}=280$. The straight line is proportional to $r^{2 / 3}$.

$$
\begin{aligned}
& \lambda=\lim _{r \rightarrow \infty} \frac{1}{\langle T(r)\rangle} \log _{10}(\rho), \\
& g^{*}=\frac{143}{81} \frac{\left(\rho^{2 / 3}-1\right)^{3}}{\rho^{2}} \frac{r^{2}}{\langle T(r)\rangle^{3}} .
\end{aligned}
$$

Results are given for the cases of forwards and backwards dispersion. Results for the case of forwards dispersion agree well with Ref. 14. From Fig. 11 we observe that the ratio for the Richardson constant for backwards and forwards dispersion, as derived from exit times is $g_{b}^{*} / g_{f}^{*} \approx 1.8$, i.e., almost equal to $g_{b} / g_{f}$, but now with $g_{b}^{*} \approx 0.8$ and $g_{f}^{*} \approx 0.45$. In Sawford, ${ }^{60}$ an explanation for this discrepancy is given. Saw-

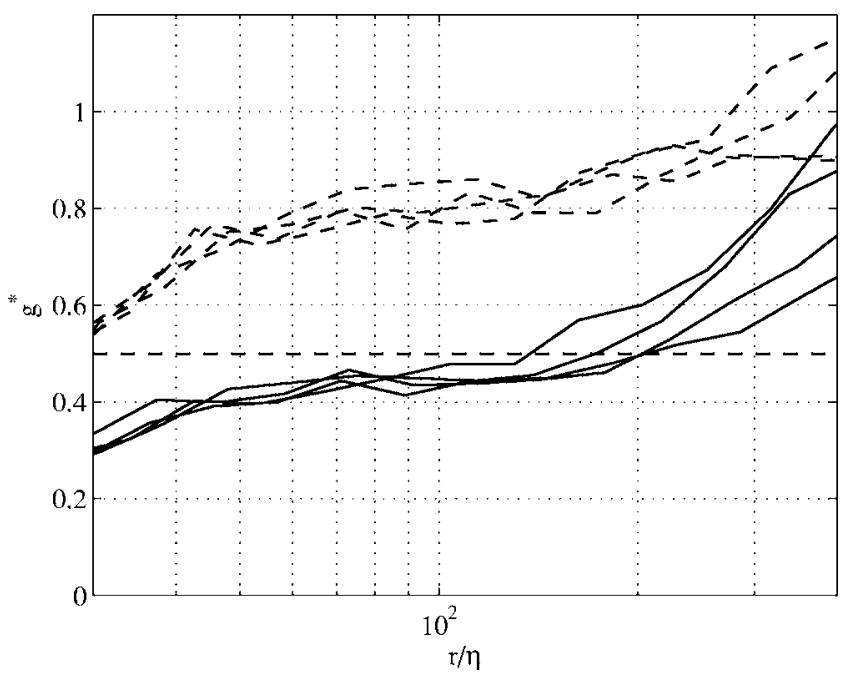

FIG. 11. Model predictions for the Richardson constant as obtained from Eq. (23), for forwards and backwards dispersion with $\rho=1.25$, dashed lines are for the backwards case $\operatorname{Re}_{\lambda}=280$; initial separations $r_{0} / \eta$ $=1.2,2.5,10,20$. The straight dashed line denotes the result obtained by Biferale et al. (Ref. 14); $g^{*} \approx 0.5$. 


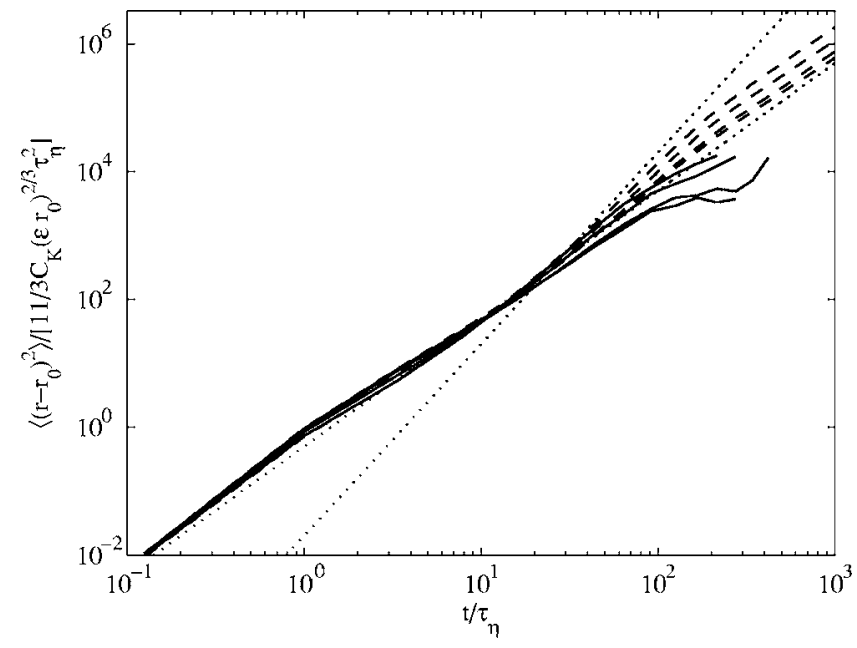

FIG. 12. Model predictions for $\left\langle\left(r-r_{0}\right)^{2}\right\rangle$ normalized with $(11 / 3) C_{k}\left(\varepsilon r_{0}\right)^{2 / 3} \tau_{\eta}^{2}$ vs time for initial separations: $r_{0} / \eta=40,80,150,200$, top to bottom. Experimental conditions of Ref. 15 are mimicked with $\mathcal{L} / \eta$ $=3000 \Rightarrow \operatorname{Re}_{\lambda}=815, d=50 \mathrm{~mm}$ (solid lines). Results for the same Reynolds number but with $d=\infty$ are plotted with dashed lines. Straight lines are proportional to $t^{2}$ and $t^{3}$.

ford argues that the way the Richardson constant is obtained from exit times, the memory of the flow is essentially neglected. From his quasi-1D model for particle dispersion, he estimates that $g_{f}$ can be underestimated by $30 \%$ and that the estimate for $g_{b}$ can easily be $50 \%$ too small. This is reflecting that (i) the flow does have a memory and (ii) that the flow memory is longer in the backwards dispersion case. From results in Ref. 60, which are derived from first-order exit time moments, it follows that $g_{b} / g_{f} \geqslant g_{b}^{*} / g_{f}^{*}$.

\section{Particle tracking and model, $\mathbf{R e}_{\lambda}=815$}

Recently, the currently highest Reynolds number particle tracking experiment performed has been reported by Bourgoin et al. ${ }^{15}$ Here, particle separation has been successfully measured in an $\operatorname{Re}_{\lambda}=815$ flow. Initial particle separations are in the range $40 \leqslant r_{0} / \eta \leqslant 1300$. The scale separation is $\mathcal{L} / \eta$ $=3000$, with an integral scale $\mathcal{L}=70 \mathrm{~mm}, \mathcal{T} / \tau_{\eta}=208$, and an observation domain of $d=50 \mathrm{~mm}$. In Ref. 15 , it is found that the ballistic regime, where particles separate as $\left\langle r^{2}(t)\right\rangle \propto t^{2}$, is valid for times smaller than $\tau_{\mathrm{B}}$, as was predicted by Batchelor. ${ }^{17}$ No transition to the Richardson-Obukhov regime was observed.

With the same parameters, we attempt to mimic the $\operatorname{Re}_{\lambda}=815$ case with the presented model (Fig. 12). For clarity, the plotted initial separations are kept to $r_{0} / \eta$ $=40,80,150,200$, since no different separation behaviors with $r_{0} / \eta>200$ can be observed. We find the modeled results to agree well with the experimental findings of Ref. 15, as all the separations follow the $\propto t^{2}$ line that was predicted by Batchelor; ${ }^{17}$ he proposed that for $t<\tau_{\mathrm{B}}$,

$$
\left\langle\left(r-r_{0}\right)^{2}\right\rangle=\frac{11}{3} C_{k}\left(\varepsilon r_{0}\right)^{2 / 3} t^{2} .
$$

To see the influence of the rather small field of view, i.e., $d / \mathcal{L} \approx 1$, we show with dashed lines the evolution of separa-
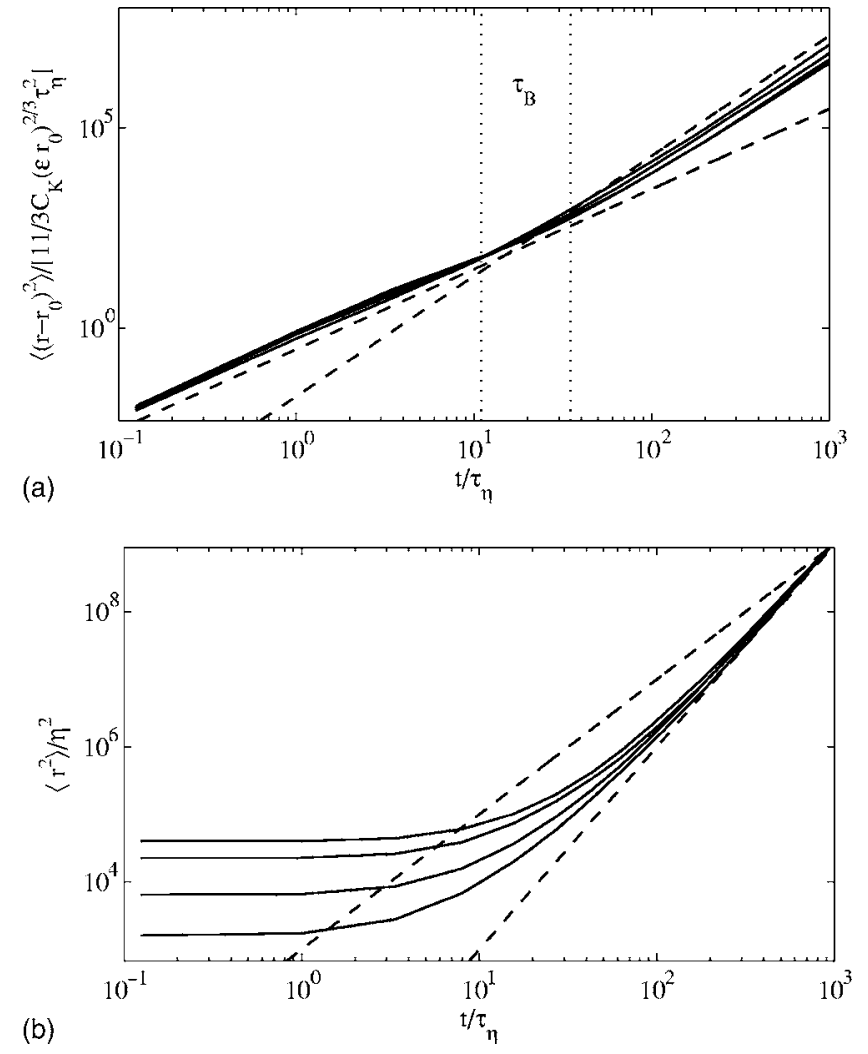

FIG. 13. Results for the ideal case with $\operatorname{Re}_{\lambda} \rightarrow \infty$ and $d=\infty$ for initial separations $r_{0} / \eta=40,80,150,200$. Straight dashed lines are proportional to $t^{2}$ and $t^{3}$; vertical dotted lines denote the range of $\tau_{\mathrm{B}}$ for the given separations. (a) Separation is plotted as in Ref. 15; dotted lines denote the range of $\tau_{\mathrm{B}}$. (b) Identical data as in (a), but plotted as in Ref. 14.

tions for the same $\operatorname{Re}_{\lambda}$ but without the finite domain effect. After their corresponding Batchelor times $\tau_{\mathrm{B}} / \tau_{\eta}$ $=11,19,28,34$ for the given $r_{0}$, we observe how the separations become faster than $\propto t^{2}$ until $r \approx \mathcal{O}(\mathcal{L})$. This is especially true for separations with $r_{0} / \eta<100$, i.e., $r_{0} / \mathcal{L}$ $<1 / 30$, as can be seen from Fig. 12. Note that $r_{0} / \mathcal{L}$ $<1 / 30$ is equivalent to $\mathcal{T} / \tau_{\mathrm{B}}>10$, since $t \sim r^{2 / 3}$, and that via a different way we arrive at the same result as Ref. 15. It thus seems that here the finite field of view is not negligible. Precisely at the time at which the beginning of a RichardsonObukhov regime may occur, particles leave the observation domain. Future experiments will tell if this prediction can actually be observed in real flows.

\section{Model only, $\operatorname{Re}_{\lambda} \rightarrow \infty$}

Like Heppe ${ }^{28}$ with his stochastic model and, e.g., Vassilicos and co-workers ${ }^{9,25,61}$ or Nicolleau and $\mathrm{Yu}^{50}$ with $\mathrm{KS}$, we check what our model predicts for the case of $\operatorname{Re}_{\lambda} \rightarrow \infty$. In Fig. 13 we show results for the same initial separations $r_{0}$ as in the $\operatorname{Re}_{\lambda}=815$ case above, but now for the ideal case of $\operatorname{Re}_{\lambda} \rightarrow \infty$ and $d=\infty$. The transition between a ballistic regime with $\left\langle r^{2}(t)\right\rangle \propto t^{2}$ and a $\left\langle r^{2}(t)\right\rangle \propto t^{3}$ regime is clearly visible. In Fig. 13(a) we see that this transition occurs at around $t=\tau_{\mathrm{B}}$. In Fig. 13(b) we observe how at late times indeed the influence of initial conditions starts to vanish and together with the $t^{3}$ slope, we can thus speak of a true RichardsonObukhov regime. This is consistent with Refs. 28 and 50. 


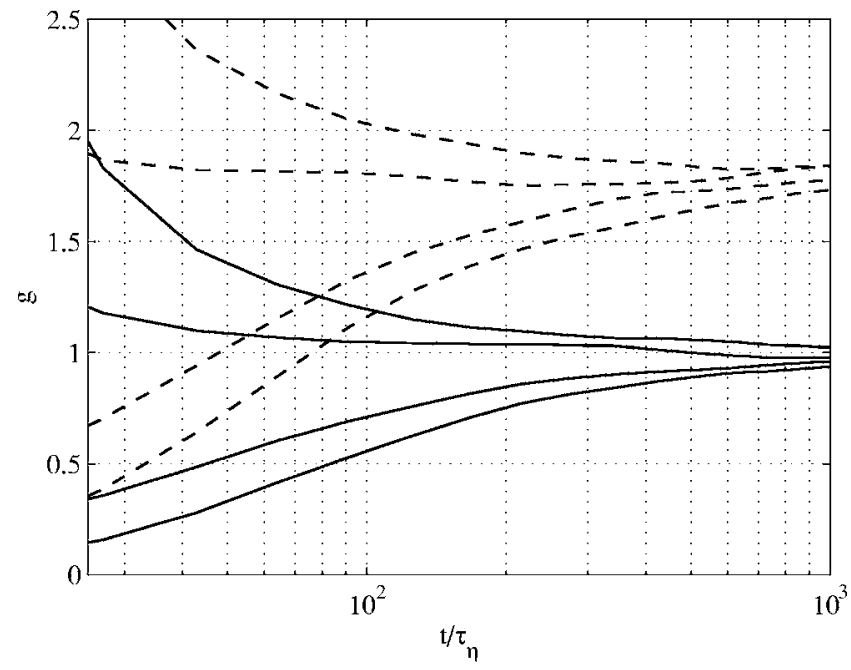

FIG. 14. Model predictions for $g=\left\langle r(t)^{2}\right\rangle /\left(\varepsilon t^{3}\right)$ without inertial range constraint, i.e., $\operatorname{Re}_{\lambda} \rightarrow \infty$, and with infinitely large observation domain, i.e., $r_{0} / \eta=1.2,2.5,10,20$. Results are given for forwards and backwards dispersion; dashed lines are for the backwards case.

We can now ask to what value and how fast the Richardson constant $g=\left\langle r^{2}\right\rangle /\left(\varepsilon t^{3}\right)$ converges for $\operatorname{Re}_{\lambda}=\infty$. From Fig. 14 we see that after a time long enough for a set of $r$ to evolve and reach its asymptotic state, the Richardson constant $g$ approaches a constant value for both the forwards and backwards dispersion cases. These values are approximately $g_{f} \approx 0.95$ and $g_{b} \approx 1.8$; i.e., $g_{b} / g_{f} \approx 1.9$. The qualitative behavior is similar to Nicolleau and Vassilicos, ${ }^{61}$ who also found a slow convergence to a such determined $g$.

To see more clearly how the distribution of $r$ reaches this asymptotic state, we show in Fig. 15 the evolution in time of the skewness, i.e., $S(t)=\left\langle[r(t)-\overline{r(t)}]^{3}\right\rangle /\left[\sigma_{r}^{2}(t)\right]^{3 / 2}$, and kurtosis, i.e., $K(t)=\left\langle[r(t)-\overline{r(t)}]^{4}\right\rangle /\left[\sigma_{r}^{2}(t)\right]^{2}$, coefficients for the same initial separations as in Fig. 14. After a transition time, both distribution parameters assume constant values, which are close but slightly below those that one obtains from the Richardson diffusion equation $\left(S_{\mathrm{R}}=1.7\right.$ and $\left.K_{\mathrm{R}}=7.8\right)$. Such a stable regime was also found in Ref. 28 which reports values slightly larger than Gaussian. The peaking behavior for the smallest separations $r_{0} / \eta=1.2$ is with $S_{p} \sim 4$ and $K_{p} \sim 30$, slightly less extreme than those measured in DNS by Biferale et al., ${ }^{14}$ who report $S_{p} \sim 4.5$ and $K_{p} \sim 40$ for $r_{0} / \eta$ $=1.2$. Yeung ${ }^{10}$ has already showed such extreme values for flatness coefficients for relative velocities. This issue that LSM models tend to underestimate flatness values was already reported in Ref. 28, where Heppe was able to match DNS results only with unphysically high values for the Lagrangian structure function constant $C_{0}$. The Borgas and Yeung $^{27}$ model, which includes viscous effects, finds a better agreement with DNS for the evolution of skewness and flatness parameters. In particular, they reproduce the more intense and earlier peaking for higher Reynolds number that is reported in Yeung and Borgas. ${ }^{62} \mathrm{KS}$ is capable to reproduce flatness parameters very well. In Malik and Vassilicos ${ }^{9}$ it was further tested if the peaking time $t_{p}$ scales with $\tau_{\eta}$ or with $\mathcal{T}$. While no clear answer was found it was concluded that $t_{p}$ has a lower bound of $\tau_{\eta}$ and an upper bound of $\mathcal{T} / 8$.

We attribute the observed peaking of distribution parameters to the exponential stretching nature in the viscous regime: Here, $d r^{2} / d t \propto r^{2}$ amplify separation rate differences much more than in the inertial range, where we have only $d r^{2} / d t \propto r^{4 / 3}$. Situations with lower relative velocities persist for a shorter time than fast separating pairs. In other words, a pair with a history of slow separation has "more chances" to change to a faster regime than an already fast separating pair has to change to a slower regime. This leads to less extreme distributions of $r$ in the inertial regime as compared to the viscous regime.

Finally, in Fig. 16 we observe in what way, according to our model, the Richardson constant is a function of Reynolds number. For runs with $r_{0} / \eta=50$ and $d=\infty$ for various $\operatorname{Re}_{\lambda}$ the Richardson constant is extracted by fitting function (1) to

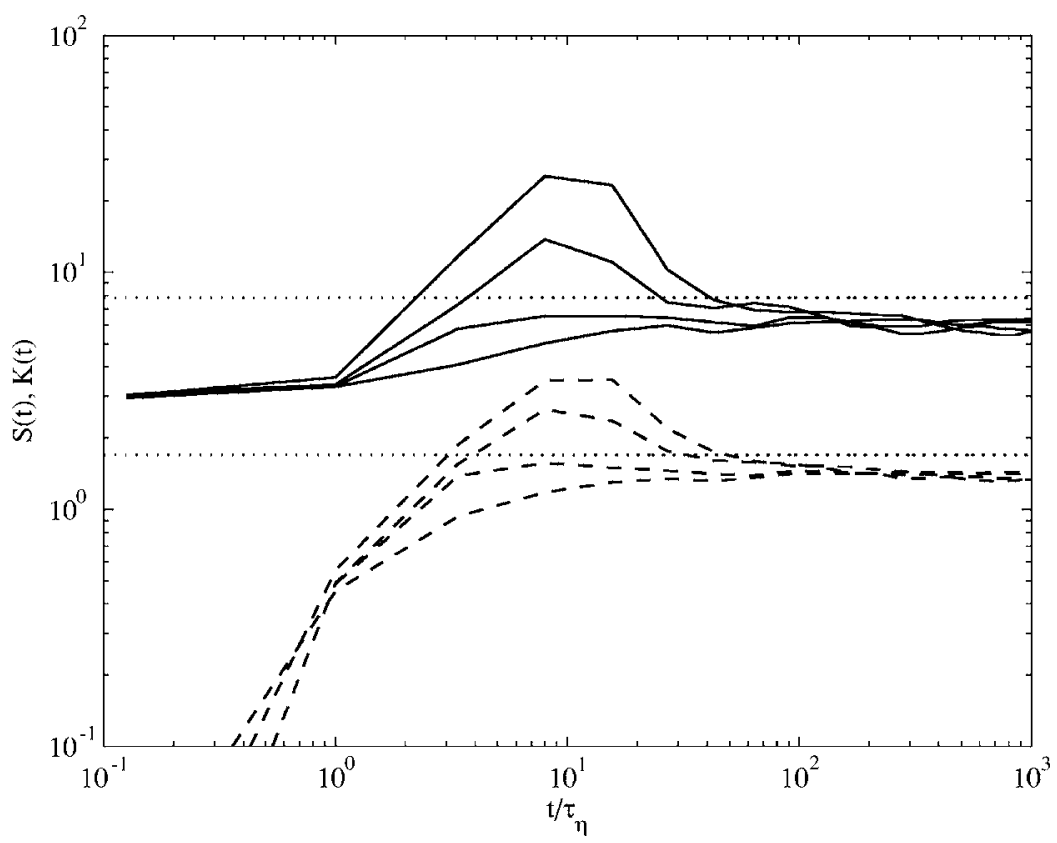

FIG. 15. Model predictions for skewness (dashed lines) and kurtosis (solid lines) coefficient of $r$ without inertial range constraint. Initial separations from top to bottom are $r_{0} / \eta=1.2,2.5,10,20$. Dotted lines denote $S_{\mathrm{R}}=1.7$ and $K_{\mathrm{R}}=7.8$ as obtained from the Richardson diffusion equation. 


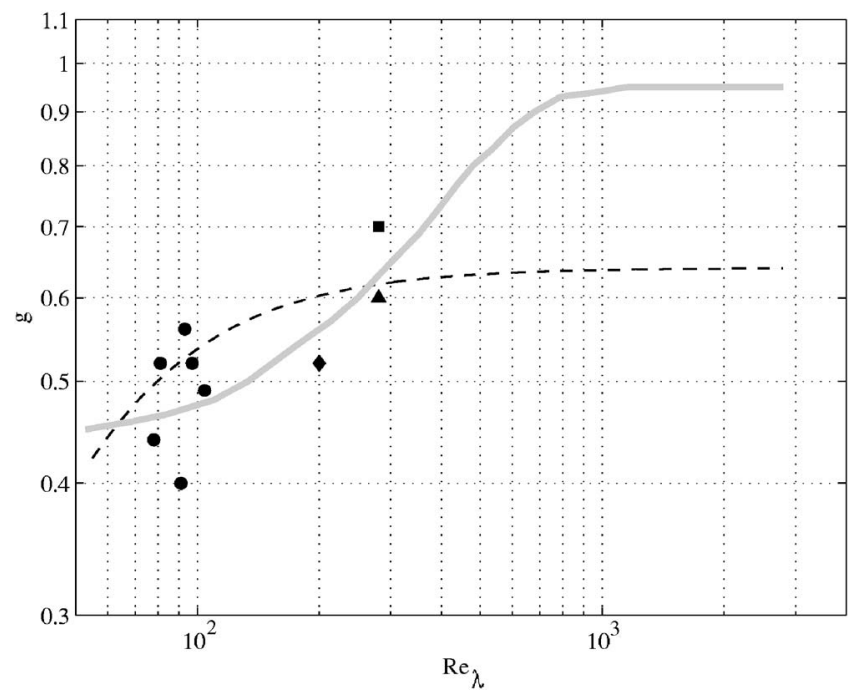

FIG. 16. Richardson constant $g$ vs $\operatorname{Re}_{\lambda}$ as obtained from the presented model, $r_{0} / \eta=50$ and $d=\infty$ (thick solid line). The solution of Franzese and Cassiani $^{19}$ is denoted as a dashed line. Circles: Ott and Mann (Ref. 11); diamond: Boffetta and Sokolov (Ref. 13); square: Ishihara and Kaneda (Ref. 12); triangle: Berg et al. (Ref. 21); as obtained from data in Ref. 14.

modeled separations, up to times before the mean separation starts to be influenced by the end of the inertial regime, as is done in Ref. 21. This procedure may be questionable for low Reynolds numbers, i.e., $\operatorname{Re}_{\lambda}<\mathcal{O}(100)$, but yields clear results as $\operatorname{Re}_{\lambda}$ increases. From Fig. 16 it appears that $g$ increases with $\operatorname{Re}_{\lambda}$ and that at $\operatorname{Re}_{\lambda} \geqslant \mathcal{O}(1000)$, an asymptotic value of $g \approx 0.95$ is approached. This result is consistent with Yeung ${ }^{63}$ who, based on extrapolated DNS data, inferred that $\operatorname{Re}_{\lambda}$ should be as high as 600-700 for a fully developed Lagrangian inertial range. It is also similar to Davila and Vassilicos ${ }^{25}$ in the sense that they report a saturation for $g$ at $\mathcal{L} / \eta>10^{3}$; however, for Davila and Vassilicos, $g \sim \mathcal{O}(0.01)$. While the qualitative behavior is similar our value is slightly higher than the result of Franzese and Cassiani ${ }^{19}$ who, after $\operatorname{Re}_{\lambda}>300$, suggest $g=0.64$. Further, the asymptotic value of $g_{f}$ is in good agreement with experimental and DNS investigations, ${ }^{10-14,21}$ slightly higher than what Ref. 28 finds and lower than results of EDQNM $(1.4<g<3.5),{ }^{22,23,38}$ LHDI $(g=5.5),{ }^{11}$ and SPH $(g=6.9) .{ }^{26}$

\section{SUMMARY AND CONCLUSION}

In this paper we have introduced a new Lagrangian stochastic model (LSM) to mimic the process of two-particle separation. The model combines a 1D Ornstein-Uhlenbeck process with a Lagrangian time scale that is a function of the evolving separation $r(t)$. A comparison with DNS and experimental results yields good agreement. Different from other LSMs, ours does not need Eulerian flow statistics. The model assumes that material line stretching in a modified form occurs also in the inertial range. The separation vector $\mathbf{r}(t)$ can be thought of as part of a coarse grained material line that is stretched by a coarse grained straining field. From K41-type arguments, it follows that the involved time scale becomes larger with increasing $r(t)$ and that for $\eta \ll r(t) \ll \mathcal{L}$ time should be $\propto \varepsilon^{-1 / 3} r^{2 / 3}$. We show that with a parametrized form of the second-order structure function, which defines the time scale for the stretching process, and the well-mixed condition the model is governed by only one function: $\bar{l}(r)$. This function can be described with one "free" parameter for each of the viscous, inertial, and diffusive regimes. The model results fit well with experimental and DNS data reported by several authors. We present results for separation distributions measured at fixed times as well as exit time statistics.

We show results for the case of backwards dispersion. In agreement with Berg et al., ${ }^{21}$ we find that the ratio of the Richardson constant for forwards and backwards dispersion is $g_{b} / g_{f} \approx 2$ for intermediate Reynolds numbers. As $\operatorname{Re}_{\lambda}$ $\rightarrow \infty$, our model predicts that $g_{b} / g_{f} \rightarrow 1.9$. If derived from exit times, we obtain $g_{b}^{*} / g_{f}^{*} \approx 1.8$, which is consistent with Sawford, ${ }^{60}$ who suggests that $\left(g_{b}^{*} / g_{f}^{*}\right)<g_{b} / g_{f}$. However, the ratio is clearly lower than the values $g_{b} / g_{f} \approx 5-20$ proposed in Ref. 59. Sawford et al. ${ }^{59}$ noted that neither LHDI nor KS are likely to differentiate between backwards and forwards dispersion, due to their inherent time reversibility for pairs and particle motion.

The model is used to explore the influence of finite observation domains. Our conclusion is that in order to observe a transition from the Batchelor regime, i.e., $\left\langle r^{2}(t)\right\rangle \propto t^{2}$, to the true Richardson-Obukhov regime, i.e., $\left\langle r^{2}(t)\right\rangle=g \varepsilon t^{3}$, both physical and experimental conditions need to be fulfilled. The physical condition is that, in addition to the flow being turbulent enough, i.e., $\operatorname{Re}_{\lambda}>\mathcal{O}(1000)$, the flow's integral scale should be large in comparison with the initial separation, $\mathcal{L} / r_{0}>30$. In addition to these "physical" conditions, we find the experimental condition that the flows integral time needs to be at least matched by the available observation time, which is defined mainly through the size of the experimental field of view. Otherwise, a transition to $\propto t^{3}$ may well occur, but cannot be observed. As a rule of thumb, we suggest that $d / \mathcal{L}>3$. We found support for these arguments from our model when we mimicked a $\operatorname{Re}_{\lambda}=815$ flow case, once for experimental conditions as in Bourgoin et al. ${ }^{15}$ and once with the finite field of view constraint removed. With an unlimited field of view, allowing for long enough observation times, the model predicts that for $r_{0} / \eta \leqslant 100$, the occurring separation just starts to tend towards $\left\langle r^{2}(t)\right\rangle \propto t^{3}$ during the time interval $\tau_{\mathrm{B}}<t<\mathcal{T}$.

Finally, we use the model to investigate the dispersion process as $\operatorname{Re}_{\lambda} \rightarrow \infty$. For small separations, we find extreme skewness and flatness coefficients at intermediate times, as was seen in Refs. 9, 10, 14, 27, and 28. For the small separations, i.e., $r_{0} / \eta \sim \mathcal{O}(1)$, presumably due to the Markov approximation $^{9,27,28}$ for the nondimensional separation rate $l(t)$ in Eq. (15), we slightly underestimate the peaking values for flatness. We attribute the peaking phenomena to a viscous process governed by exponential stretching. At later times, the modeled separation distributions are in fair agreement with the Richardson diffusion equation and $\left\langle r^{2}(t)\right\rangle=g \varepsilon t^{3}$, as it is reported in Refs. 28 and 50. Further, we find that for high Reynolds numbers, i.e., $\operatorname{Re}_{\lambda}>\mathcal{O}(1000)$, times $t / \tau_{\eta}$ $\approx \mathcal{O}(1000)$ are necessary for the Richardson constant to converge to an asymptotic value of $g_{f} \approx 0.95$ and $g_{b} \approx 1.8$, for forwards and backwards dispersion, respectively. Our values 
for $g_{f}$ are in good agreement with experimental and DNS investigations ${ }^{10-14,21}$ and the theory of Ref. 19 for the still limited range for which data are available. Our $g_{f}$ is higher than what Ref. 28 finds and considerably higher than values stemming from KS studies. ${ }^{25}$ On the other hand, $1.4<g$ $<3.5$ from EDQNM, ${ }^{22,23,38} g=5.5$ from LHDI, ${ }^{11}$ and $g=6.9$ from $\mathrm{SPH}^{26}$ are reported. The transition of $0.5 \leqslant g \leqslant 0.95$ occurs over a range of Reynolds number from $\mathcal{O}(100) \leqslant \operatorname{Re}_{\lambda}$ $\leqslant \mathcal{O}(1000)$. The asymptotic behavior is consistent with the theoretical result of Ref. 19.

The model presented seems to work satisfactorily and is consistent with a variety of experimental and DNS investigations. The model is simple in the sense that it reduces the complex separation dynamics to one single time scale, which is defined by the separation $r(t)$ itself. Further, the model requires information neither on Eulerian acceleration statistics nor on the shape of the Eulerian velocity PDF. The time scale that is governed by the evolving $r(t)$ is essentially making the model a Lagrangian one. The good results suggest that a Gaussian process combined with "Lagrangianity" is sufficient to capture the essential features of the relative particle dispersion problem. To what degree our results are also obtained for the right reasons, future experiments will have to show, e.g., the alignment of the separation vector $\mathbf{r}(t)$ to the corresponding coarse grained strain field will have be measured. Similarly, our findings on the influence of finite observation domain and on the precise value for the Richardson constant need to be checked by experiment.

\section{ACKNOWLEDGMENTS}

Part of this work was financed by the EU project "Fluid Mechanical Stirring and Mixing: the Lagrangian Approach," coordinated by A. Pumir. Further, we gratefully acknowledge grants of the Danish Research Agency (274-05-0540) and of the Swiss Science Foundation (PA0024-4111444/1). It is our pleasure to mention helpful comments and discussions with A. Liberzon and M. Guala.

${ }^{1}$ L. F. Richardson, "Atmospheric diffusion shown on a distance-neighbour graph,” Proc. R. Soc. London, Ser. A 110, 709 (1926).

${ }^{2}$ B. Sawford, "Turbulent relative dispersion," Annu. Rev. Fluid Mech. 33, 289 (2001).

${ }^{3}$ J. S. Shirolkar, C. F. M. Coimbra, and M. Q. McQuay, "Fundamental aspects of modeling turbulent particle dispersion in dilute flows," Prog. Energy Combust. Sci. 22, 363 (1996).

${ }^{4}$ J. Mann, S. Ott, H. L. Pecseli, and J. Trulsen, "Predator-prey encounters in turbulent waters," Phys. Rev. E 65, 026304 (2002).

${ }^{5}$ J. Mann, S. Ott, H. L. Pecseli, and J. Trulsen, "Turbulent particle flux to a perfectly absorbing surface," J. Fluid Mech. 534, 1 (2005).

${ }^{6}$ J. Berg Jørgensen, J. Mann, S. Ott, H. L. Pecseli, and J. Trulsen, "Experimental studies of occupation and transit times in turbulent flows," Phys. Fluids 17, 035111 (2005).

${ }^{7}$ J. C. H. Fung, J. C. R. Hunt, N. A. Malik, and R. J. Perkins, "Kinematic simulation of homogeneous turbulence by unsteady random Fourier modes," J. Fluid Mech. 236, 281 (1992).

${ }^{8}$ J. C. H. Fung and J. C. Vassilicos, "Two-particle dispersion in turbulentlike flows," Phys. Rev. E 57, 1677 (1998).

${ }^{9}$ N. A. Malik and J. C. Vassilicos, "A Lagrangian model for turbulent dispersion with turbulent-like flow structure: Comparison with direct numerical simulation for two-particle statistics," Phys. Fluids 11, 1572 (1999).

${ }^{10} \mathrm{P}$. K. Yeung, "Direct numerical simulation of two-particle relative diffusion in isotropic turbulence," Phys. Fluids 6, 3416 (1994).

${ }^{11} \mathrm{~S}$. Ott and J. Mann, "An experimental investigation of the relative diffu- sion of particle pairs in three dimensional turbulent flow," J. Fluid Mech. 422, 207 (2000).

${ }^{12} \mathrm{~T}$. Ishihara and Y. Kaneda, "Relative diffusion of a pair of fluid particles in the inertial subrange of turbulence," Phys. Fluids 14, L69 (2002).

${ }^{13}$ G. Boffetta and I. M. Sokolov, "Relative dispersion in fully developed turbulence: The richardson's law and intermittency corrections," Phys. Rev. Lett. 88, 094501 (2002).

${ }^{14}$ L. Biferale, G. Boffetta, A. Celani, B. J. Devenish, A. Lanotte, and F. Toschi, "Lagrangian statistics of particle pairs in homogeneous isotropic turbulence," Phys. Fluids 17, 115101 (2005).

${ }^{15}$ M. Bourgoin, N. T. Ouellette, H. Xu, J. Berg, and E. Bodenschatz, "Pair dispersion in turbulence," Science 311, 835 (2006).

${ }^{16}$ A. M. Obukhov, "Spectral energy distribution in turbulent flow," Dokl. Akad. Nauk SSSR 5, 453 (1941)

${ }^{17}$ G. K. Batchelor, "Diffusion in a field of homogeneous turbulence. II The relative motion of particles," Proc. Cambridge Philos. Soc. 48, 345 (1952).

${ }^{18}$ R. H. Kraichnan, "Dispersion of particle pairs in homogeneous turbulence," Phys. Fluids 10, 1937 (1966).

${ }^{19}$ P. Franzese and M. Cassiani, "A statistical theory of turbulent relative dispersion,” J. Fluid Mech. 571, 391 (2007).

${ }^{20}$ G. Falkovich, K. Gawedzki, and M. Vergassola, "Particles and fields in fluid turbulence," Rev. Mod. Phys. 73, 913 (2001).

${ }^{21}$ J. Berg, B. Lüthi, J. Mann, and S. Ott, "An experimental investigation: backwards and forwards relative dispersion in turbulent flow," Phys. Rev. E 74, 016304 (2006).

${ }^{22}$ Ye. A. Novikov, "Random force method in turbulence theory," Sov. Phys. JETP 17, 1449 (1963).

${ }^{23}$ M. Larchevêque and M. Lesieur, "The application of eddy-damped Markovian closures to the problem of dispersion of particle pairs," J. Mec. 20, 113 (1981).

${ }^{24}$ D. R. Osborne, J. C. Vassilicos, K. Sung, and J. D. Haigh, "Fundamentals of pair diffusion in kinematic simulations of turbulence," Phys. Rev. E 74, 036309 (2006)

${ }^{25}$ J. Davila and J. C. Vassilicos, "Richardson's pair diffusion and the stagnation point structure of turbulence," Phys. Rev. Lett. 91, 1 (2003).

${ }^{26} \mathrm{~S}$. Goto and J. C. Vassilicos, "Particle pair diffusion and persistent streamline topology in two-dimensional turbulence," New J. Phys. 65, 1 (2004).

${ }^{27}$ M. S. Borgas and P. K. Yeung, "Relative dispersion in isotropic turbulence. Part 2. A new stochastic model with Reynolds-number dependence," J. Fluid Mech. 503, 125 (2004).

${ }^{28}$ B. M. O. Heppe, "Generalized Langevin equation for relative turbulent dispersion," J. Fluid Mech. 357, 167 (1998).

${ }^{29}$ G. Pedrizzetti and E. A. Novikov, "On Markov modelling of turbulence," J. Fluid Mech. 280, 69 (1994).

${ }^{30}$ D. J. Thomson, "A stochastic model for the motion of particles pairs in isotropic high-Reynolds-number turbulence, and its application to the problem of concentration variance," J. Fluid Mech. 210, 113 (1990).

${ }^{31}$ P. A. Durbin, "A stochastic model of two-particle dispersion and concentration fluctuations in homogeneous turbulence," J. Fluid Mech. 100, 279 (1980).

${ }^{32}$ N. Mordant, J. Delour, E. Léveque, A. Arnéodo, and J.-F. Pinton, "Long time correlations in Lagrangian dynamics: A key to intermittency in turbulence," Phys. Rev. Lett. 89, 1 (2002).

${ }^{33}$ G. A. Voth, A. La Porta, A. M. Crawford, J. Alexander, and Eberhard Bodenschatz, "Measurements of particle accelerations in fully developed turbulence," J. Fluid Mech. 469, 121 (2002).

${ }^{34}$ A. La Porta, G. A. Voth, A. M. Crawford, J. Alexander, and E. Bodenschatz, "Fluid particle accelerations in fully developed turbulence," Nature 409, 1016 (2001).

${ }^{35} \mathrm{~V}$. I. Tatarski, "Radiophysical methods of investigating atmospheric turbulence," Izv. Vyssh. Uchebn. Zaved., Radiofiz. 5, 551 (1960).

${ }^{36}$ N. L. Byzova, Y. K. Garger, and V. N. Ivanov, "Experimental estimation of the Lagrangian time scale of turbulence," Izv., Acad. Sci., USSR, Atmos. Oceanic Phys. 6, 315 (1970).

${ }^{37}$ V. Artale, G. Boffetta, A. Celani, M. Cencini, and A. Vulpiani, "Dispersion of passive tracers in closed basins: beyond the diffusion coefficient," Phys. Fluids 9, 3162 (1997).

${ }^{38}$ David J. Thomson, "The separation of particle pairs in the eddy-damped quasinormal Markovian approximation," Phys. Fluids 8, 642 (1996).

${ }^{39}$ D. J. Thomson and B. J. Devenish, "Particle pair separation in kinematic simulations," J. Fluid Mech. 526, 277 (2005).

${ }^{40}$ M.-C. Jullien, J. Paret, and P. Tabeling, "Richardson pair dispersion in two-dimensional turbulence," Phys. Rev. Lett. 82, 2872 (1999). 
${ }^{41}$ M. S. Borgas and B. L. Sawford, "A family of stochastic models for two-particle dispersion in isotropic homogeneous stationary turbulence," J. Fluid Mech. 279, 69 (1994).

${ }^{42}$ O. A. Kurbanmuradov, "A new Lagrangian model of two-particle relative turbulent dispersion,” Monte Carlo Meth. Appl. 1, 83 (1995).

${ }^{43}$ P. K. Yeung, "One- and two-particle Lagrangian acceleration correlations in numerically simulated homogeneous turbulence,” Phys. Fluids 9, 2981 (1997).

${ }^{44}$ U. Frisch, Turbulence: The Legacy of A. N. Kolmogorov (Cambridge University Press, Cambridge, UK,1995).

${ }^{45}$ A. Naso and A. Pumir, "Scale dependence of the coarse-grained velocity derivative tensor structure in turbulence," Phys. Rev. E 72, 056318 (2005).

${ }^{46}$ V. Borue and S. A. Orzag, "Local energy flux and subgrid-scale statistics in three-dimensional turbulence," J. Fluid Mech. 366, 1 (1998).

${ }^{47}$ B. Tao, J. Katz, and C. Meneveau, "Statistical geometry of subgrid-scale stresses determined from holographic particle image velocimetry measurements," J. Fluid Mech. 457, 35 (2002).

${ }^{48}$ A. Tsinober, An Informal Introduction to Turbulence (Kluwer Academic, Dordrecht, 2001).

${ }^{49}$ G. K. Batchelor, "The application of the similarity theory of turbulence to atmospheric diffusion," Q. J. R. Meteorol. Soc. 76, 133 (1950).

${ }^{50}$ F. Nicolleau and G. Yu, "Turbulent pair diffusion," Phys. Rev. Lett. 90, 1 (2004).

${ }^{51}$ S. S. Girimaji and S. B. Pope, "Material-element deformation in isotropic turbulence," J. Fluid Mech. 220, 427 (1990).

${ }^{52}$ E. Dresselhaus and M. Tabor, "The kinematics of stretching and alignment of material elements in general flow fields," J. Fluid Mech. 236, 415 (1994).

${ }^{53}$ M.-J. Huang, "Correlations of vorticity and material line elements with strain in decaying turbulence," Phys. Fluids 8, 2203 (1996).

${ }^{54}$ B. Lüthi, A. Tsinober, and W. Kinzelbach, "Lagrangian measurement of vorticity dynamics in turbulent flow," J. Fluid Mech. 528, 87 (2005).

${ }^{55}$ M. Guala, B. Lüthi, A. Liberzon, A. Tsinober, and W. Kinzelbach, "On the evolution of material lines and vorticity in homogeneous turbulence," J. Fluid Mech. 533, 339 (2005).

${ }^{56} \mathrm{G}$. K. Batchelor, "The effect of homogeneous turbulence on material lines and surfaces," Proc. R. Soc. London 213, 349 (1952).

${ }^{57}$ B. L. Sawford, "Reynolds number effects in Lagrangian stochastic models of turbulent dispersion," Phys. Fluids A 3, 1577 (1991).

${ }^{58}$ C. W. Gardiner, Handbook of Stochastic Methods (Springer Verlag, Berlin, 1985).

${ }^{59}$ B. Sawford, P. K. Yeung, and M. S. Borgas, "Comparison of backwards and forwards relative dispersion in turbulence," Phys. Fluids 17, 095109 (2005).

${ }^{60}$ B. Sawford, "A study of the connection between exit-time statistics and relative dispersion using a simple Lagrangian stochastic model," J. Turbul. 7, 1 (2006).

${ }^{61}$ F. Nicolleau and J. C. Vassilicos, "Two-particle diffusion and locality assumption," Phys. Fluids 16, 2309 (2003).

${ }^{62}$ P. K. Yeung and M. S. Borgas, "Relative dispersion in isotropic turbulence. Part 1. Direct numerical simulations and Reynolds-number dependence," J. Fluid Mech. 503, 93 (2004).

${ }^{63}$ P. K. Yeung, "Lagrangian investigations of turbulence," Annu. Rev. Fluid Mech. 34, 115 (2002). 